\title{
Interactions between Borrelia burgdorferi and ticks
}

\section{Cheyne Kurokawa ${ }^{1,6}$, Geoffrey E. Lynn (1) ${ }^{1,6}$, Joao H. F. Pedra ${ }^{2}{ }^{2}$, Utpal Pal ${ }^{3,4}$, Sukanya Narasimhan (1) ' and Erol Fikrig (10) 1,5凶}

\begin{abstract}
Borrelia burgdorferi is the causative agent of Lyme disease and is transmitted to vertebrate hosts by lxodes spp. ticks. The spirochaete relies heavily on its arthropod host for basic metabolic functions and has developed complex interactions with ticks to successfully colonize, persist and, at the optimal time, exit the tick. For example, proteins shield spirochaetes from immune factors in the bloodmeal and facilitate the transition between vertebrate and arthropod environments. On infection, B. burgdorferi induces selected tick proteins that modulate the vector gut microbiota towards an environment that favours colonization by the spirochaete. Additionally, the recent sequencing of the Ixodes scapularis genome and characterization of tick immune defence pathways, such as the JAK-STAT, immune deficiency and cross-species interferon- $\gamma$ pathways, have advanced our understanding of factors that are important for B. burgdorferi persistence in the tick. In this Review, we summarize interactions between B. burgdorferi and I. scapularis during infection, as well as interactions with tick gut and salivary gland proteins important for establishing infection and transmission to the vertebrate host.
\end{abstract}

${ }^{1}$ Section of Infectious Diseases, Department of Internal Medicine, Yale University School of Medicine, New Haven, CT, USA.

${ }^{2}$ Department of Microbiology and Immunology, University of Maryland School of Medicine, Baltimore, MD, USA.

${ }^{3}$ Department of Veterinary Medicine, University of Maryland, College Park, MD, USA.

${ }^{4}$ Virginia-Maryland Regional College of Veterinary Medicine, College Park, MD, USA.

${ }^{5}$ Howard Hughes Medical Institute, Chevy Chase, MD, USA.

${ }^{6}$ These authors contributed equally: Cheyne Kurokawa, Geoffrey E. Lynn.

凶e-mail:erol.fikrig@yale.edu https://doi.org/10.1038 s41579-020-0400-5
Lyme disease is a tick-borne disease caused by the spirochaete Borrelia burgdorferi, which is transmitted enzootically between ticks and their hosts, resulting in approximately 300,000 cases annually in the United States $^{1,2}$. Globally, several species within the B. burgdorferi sensu lato complex have been identified as human pathogens, however, in the United States, nearly all Lyme disease is caused by B. burgdorferi sensu stricto (referred to as B. burgdorferi in this Review). Erythema migrans, the characteristic expanding rash, is an indicator of early acute infection, although the disease can also present with a variety of non-specific clinical signs. Spirochaetes enter the human skin at the tick bite site and then use internal periplasmic flagella to migrate to distal tissues, including the heart and joints ${ }^{3}$. Untreated infections can progress to multisystemic manifestations including rheumatologic, neurologic and cardiac disease. Similar versions of Lyme disease occur throughout the Northern Hemisphere, where Ixodes tick species are present. In Europe, Lyme borreliosis is caused by B. burgdorferi sensu lato complex spirochaetes (BOX 1), which may infect as many as 85,000 persons annually, while in Asia fewer epidemiological studies have been reported, and it is likely that the true incidence is not well understood.

The genome of B. burgdorferi consists of an approximately $1-\mathrm{Mb}$ linear chromosome and at least 17 circular and linear plasmids, many of which are highly stable and contain genes that are crucial for survival ${ }^{4,5}$ (BOX 2). Gene expression is highly regulated to enable the spirochaete to adapt to the different environments as it cycles between an arthropod host and a vertebrate host ${ }^{6}$. External cues from the host, such as temperature, $\mathrm{pH}, \mathrm{CO}_{2}$ levels and other biotic factors, as well as host species are important factors that regulate gene expression in B. burgdorferi ${ }^{7-10}$. $B$. burgdorferi undergoes several changes during transmission from the tick to the host to adapt to the new conditions. At the bite site, the spirochaete must evade the immune defences of the mammalian host to extravasate and establish infection in other tissues. Although B. burgdorferi genome encodes several proteins to facilitate these functions, it also relies heavily on interactions with tick salivary proteins injected into the bite site during the initial stage of vertebrate infection. Understanding how the spirochaetes and the tick host interact is crucial to better understand infection, pathogen transmission and potential targeted therapies.

In the United States, most tick-borne infections are transmitted by the bite of the blacklegged tick, Ixodes scapularis, including infections with B. burgdorferi, Borrelia miyamotoi, Borrelia mayonii, Babesia microti, Ehrlichia muris eauclairensis, Anaplasma phagocytophilum and Powassan virus. This three-host tick species is the primary vector for Lyme disease-causing $B$. burgdorferi spirochaetes. The life cycle of I. scapularis spans 2-4 years and includes egg, larval, nymphal and adult stages. 


\section{Box 1 | Lyme disease and Lyme borreliosis in the United States and Europe}

\section{Tick vectors}

In the United States, Ixodes scapularis is the primary tick species associated with human transmission except for the West Coast, where Ixodes pacificus is the most important vector. In Europe, Ixodes ricinus is the primary vector for human transmission, although Ixodes persulcatus is also a source of infections in certain regions ${ }^{198}$. In Asia, I. persulcatus as well as various other Ixodes species and Haemaphysalis species are vectors for Borrelia burgdorferi.

\section{Borrelia genospecies}

In the United States, B. burgdorferi sensu stricto is the aetiological agent of Lyme disease. A more recently discovered species, Borrelia mayonii, is also present in the North Central region of the United States, where it can overlap in clinical presentation with Lyme disease caused by B. burgdorferi sensu stricto, yet accounts for a much smaller number of reported human infections ${ }^{199}$. In Europe, most cases of Lyme borreliosis are caused by Borrelia afzelii, Borrelia garinii and to a much lesser extent B. burgdorferi sensu stricto and Borrelia bavariensis ${ }^{200,201}$.

\section{Estimated annual infections}

In the United States $\sim 33,000$ cases were reported in 2018 , although the number of estimated annual infections is closer to 300,000 (REFS ${ }^{1,2,202}$ ). In Europe, Lyme borreliosis is not a mandatory reportable disease in many countries. However, $\sim 85,000$ cases are reported annually, which is likely to be a severe underestimate ${ }^{203}$.

\section{Geographic distribution}

Lyme disease incidence is highest in the Northeast and North Central regions of the United States, although infections can be acquired in the West Coast and South as well ${ }^{19,202}$. In Europe, I. ricinus is widely distributed throughout the continent, and human cases are likely to be closely associated with the distribution of the vector.

\section{Hosts}

In both the United States and Europe, small mammals, especially rodents, are reservoirs for Borrelia spp. infections and are important hosts for immature Ixodes spp. stages. Deer have minimal importance as reservoirs for spirochaetes that cause Lyme disease, but as the primary hosts for adult stages, they are crucial for tick reproduction. Some birds, similarly to deer, are important sources for tick dispersal and seeding in new populations ${ }^{204}$, as well as transmission of spirochaetes.

\section{Clinical presentation}

Lyme disease (in the United States) and Lyme borreliosis (in Europe) are highly similar in their primary clinical features and may include multisystemic disease of the skin, joints, heart and nervous system. However, in the United States, systemic disease, including a rapid advancement of erythema migrans, is more common (approximately $70 \%$ of infected individuals), and in the absence of antibiotic treatments, Lyme arthritis seems to be a more likely outcome than in Europe. In Europe, neuroborreliosis is more common, acrodermatitis chronica atrophicans and borrelial lymphocytoma are reported more frequently and erythema migrans expands more slowly with greater central clearing relative to the typical presentation in the United States ${ }^{198,200}$. The clinical features of Lyme borreliosis seem to be associated with distinct genotypes and tissue tropisms of specific species of B. burgdorferi sensu lato.

\section{Enzootically \\ Describes a pathogen \\ that is maintained through \\ transmission among \\ non-human animal reservoirs.}

Three-host tick species

Ticks that leave the host after feeding during each stage of

development. This is in contrast to single-host tick species,

which remain attached to the same host from larva to adults.
Most tick-borne pathogens, including B. burgdorferi, are acquired during the larval or nymphal feed and are transmitted by nymphs or adults (FIG. 1), with the exception of transovarially acquired pathogens. Immature I. scapularis ticks are generalist feeders, parasitizing small mammals, medium-sized mammals, birds and reptiles ${ }^{11}$. Adults also feed on medium-sized mammals, although the primary host for this stage is the white-tailed deer, Odocoileus virginianus ${ }^{12}$.

I. scapularis activity patterns are highly seasonal and vary by geography ${ }^{13,14}$. Tick phenology is therefore an important factor in the epidemiology of tick-borne pathogens ${ }^{14,15}$. In the North Central region of the United States, larvae are most active during June and July, whereas larval emergence is bimodal in the Northeast, with peaks in the spring and late summer ${ }^{16,17}$. Nymphs are most active in June and July, and although adults can be active year-round under ideal conditions, they are encountered most often in spring and in autumn. The incidence of Lyme disease is greatest during the months when nymphs are most active ${ }^{18,19}$ (BOX 1). Although I. scapularis is also present in regions of the United States other than the North Central and Northeast regions, several factors, including disparate host-seeking behaviour of immature stages, result in a lower prevalence of B. burgdorferi in ticks and a lower risk of Lyme disease in these other regions ${ }^{20}$.

Whereas much of the ecology and epidemiology of B. burgdorferi and Lyme disease have been understood for decades, the sequencing of the I. scapularis genome in 2016 as well as studies examining the tick transcriptome and proteome have greatly advanced the current understanding of the tick immune defence pathways ${ }^{21-26}$. In this Review, we focus on these findings and how they have enabled researchers to better understand interactions between B. burgdorferi and I. scapularis ${ }^{22,27-30}$. We describe key interactions specific to the tick gut and highlight the influence of the tick gut microbiota, as well as mechanisms that the spirochaete uses to alter the microbiota $^{31-34}$. Lastly, we highlight tick-B. burgdorferi interactions in the salivary glands that are important for transmission to mammalian hosts.

\section{Interactions in the gut}

B. burgdorferi spirochaetes are highly motile and use periplasmic flagella to propel themselves through host fluids and tissues ${ }^{35}$. When a tick feeds on an infected vertebrate host, spirochaetes are attracted to the tick feeding site by chemotactic signals where they are ingested during the feeding process ${ }^{36}$. The tick gut is the initial site of colonization of B. burgdorferi $i^{37}$, and there the spirochaete must overcome several barriers to persist in the tick, such as evading tick immune defences ${ }^{22,29,30}$ and avoiding endocytic digestion in tick gut epithelial cells ${ }^{38}$. Most spirochaetes remain in the lumen of the tick gut for the duration of the moulting process before migrating to the salivary glands during subsequent feedings ${ }^{39}$. To facilitate colonization and persistence in the gut, B. burgdorferi has evolved elaborate mechanisms to modulate the gut environment. Importantly, B. burgdorferi accomplishes these processes with minimal fitness cost to the tick ${ }^{40}$. Here we discuss interactions between $B$. burgdorferi and the tick in the gut environment, the tick immune response to infection with $B$. burgdorferi and the mechanisms that $B$. burgdorferi uses to establish infection in the tick.

Outer surface protein interactions in the gut. The presence of spirochaetes in tick salivary glands is essential for transmission to a new vertebrate host. However, the gut is the principal tissue of residence for B. burgdorferi during most of its arthropod phase and is therefore also a key site for its interactions with the tick. In an unfed infected tick, B. burgdorferi is found closely associated with the gut epithelial cells ${ }^{37,41}$. As blood flow and temperature shift during the tick bloodmeal, spirochaetes must adjust to a reduction in $\mathrm{pH}$ from 7.4 to $6.8\left(\mathrm{REFS}^{42,43}\right)$. To rapidly adapt to changes encountered in hostile and physiologically dissimilar host 
Neuroborreliosis

Neurological manifestation

of disease that can occur as

part of systemic infection

with Borrelia spirochaetes,

including Borrelia burgdorferi.

Acrodermatitis chronica atrophicans

A late manifestation of chronic

Borrelia burgdorferi infection

characterized by blue-red skin

lesions and swelling, typically on the extremities. environments, for example in the tick gut, $B$. burgdorferi uses preferential gene expression.

A relatively well-studied example of tick-B. burgdorferi interactions involves several outer surface proteins. For successful acquisition of spirochaetes following the tick bloodmeal, the outer surface proteins OspA and OspB are important for adherence and persistent colonization of the tick gut. Binding of OspA to TROSPA, a tick gut protein upregulated during tick feeding and downregulated on repletion, contributes to this process $^{4-48}$ (FIG. 2). Whereas OspA expression facilitates establishment of $B$. burgdorferi in the gut of a previously uninfected tick, for transmission to a new vertebrate host during a subsequent bloodmeal, spirochaetes must exit

\section{Box 2 | Biology of Borrelia burgdorferi}

Borrelia burgdorferi belongs to the phylum Spirochaetes and the spirochaetes have a distinct spiral shape with a flat-wave morphology ${ }^{205}$. B. burgdorferi spirochaetes lack classic lipopolysaccharide in the outer membrane and are described as Gram-negativelike ${ }^{206}$. The spirochaetes contain both an outer lipid bilayer and an inner lipid bilayer, a compositionally distinct peptidoglycan layer with flagella in the periplasmic space between the two membranes, which protects from recognition by the host immune system ${ }^{207}$. Approximately 7 to 11 flagella are located at both ends of the spirochaete and form a ribbon that wraps around the spirochaete ${ }^{207}$. The flagella give $B$. burgdorferi its structural shape and enable motility in environments such as tick saliva and the highly viscous extracellular matrix network in the dermis of mammals ${ }^{207}$. In the skin, several immune signalling pathways, including those signalling through MyD88, have a role in controlling the initial colonization ${ }^{208}$; however, spirochaetes that can evade innate immune recognition disseminate to secondary infection sites, such as the heart, joint tissues, urinary bladder and nervous system. As B. burgdorferi lacks classic bacterial secretion apparatus and toxins, the carditis, arthritis and neuritis observed in persistently infected patients is likely caused by the inflammatory immune response at the site of infection, which can be induced by certain spirochaete antigens, including lipoproteins.

The genome of B. burgdorferi is composed of an approximately 1-Mb linear chromosome and at least 17 circular and linear plasmids ${ }^{4}$. Although the chromosome encodes many bacterial orthologues with known or housekeeping functions, the vast majority of plasmid-encoded genes are unique to Borrelia spp. and are unrelated to known proteins. The genome encodes relatively few genes involved in response to oxidative and nitrosative stress ${ }^{4,136,137}$. Additionally, B. burgdorferi encodes limited genes involved in metabolic pathways; therefore, it relies heavily on the host and uses transport systems to scavenge nutrients from the environment, such as the manganese transporter bb0219 (REFS ${ }^{80,81,209}$ ), which maintains the metabolic flexibility needed to use the different nutrients available in arthropod and vertebrate environments. In mammals, glucose is the primary source of carbon in blood ${ }^{82}$, whereas glycerol and, to a lesser extent, chitobiose are available to spirochaetes in the tick environment ${ }^{62,83-85}$. The second messenger c-di-GMP upregulates genes and induces an effector protein that enables spirochaetes to use alternative pathways of carbon metabolism ${ }^{60}$. Moreover B. burgdorferi mutants lacking the ability to use glycerol could infect mice normally yet were present at much lower levels in experimentally infected nymphs than in wild-type spirochaetes ${ }^{84}$. The genome also does not encode components of the tricarboxylic acid cycle or enzymes required for nucleotide and fatty acid synthesis ${ }^{4}$.

Much of the research on B. burgdorferi biology has focused on lipoproteins on the outer membrane, referred to as 'outer surface proteins', because of their important role in spirochaete survival, including adaptation to and navigation of physiologically and immunologically hostile host environments. Several studies have documented antigenic variation in outer surface protein expression as B. burgdorferi transitions between the tick host and the mammalian host ${ }^{10,48-50,52}$. In the mammalian host, certain outer surface proteins can activate neutrophils, B cells, T cells and dendritic cells, whereas others inhibit neutrophils, natural killer cells and complement activation ${ }^{210}$ Recent evidence demonstrated that B. burgdorferi can shield itself from immunogenic proteins with the highly variable VlsE surface protein ${ }^{211}$. The VlsE antigen is essential for persistence in the mammalian host and undergoes robust antigenic switching through recombination events in the vlsE locus and 15 silent vls cassettes ${ }^{212}$. Importantly, the high degree of heterogeneity, as well as the shielding and antigenic switching, complicates the development of an effective vaccine. the gut and pass through the salivary glands. There are contrasting reports about the mechanisms of spirochaete migration. Initial studies suggested that the incoming bloodmeal and alteration in temperature and $\mathrm{pH}$ result in downregulation of OspA and upregulation of $\mathrm{OspC}^{10,42,49}$. This led to the initial hypothesis that loss of OspA expression is associated with migration of the spirochaetes from the gut, and expression of OspC is linked with movement of $B$. burgdorferi out of the tick and the establishment of mammalian infection ${ }^{44,49,50}$. Later studies showed no reciprocal expression of OspA and OspC; instead, OspA expression was maintained throughout the feeding proces ${ }^{51-53}$. Additionally, robust OspA expression was detected in nearly all spirochaetes throughout the course of the bloodmeal and decreased only in the mammalian host ${ }^{52}$. Live microscopy has shown a biphasic process of migration in which networks of replicating non-motile spirochaetes, by adhering to differentiating, hypertrophying and detaching epithelial cells, migrate towards the basolateral surface of the gut epithelium ${ }^{41}$. In the second phase, the spirochaetes transition into motile organisms that are able to traverse the basal membrane, enter the haemocoel and migrate to salivary glands ${ }^{41}$.

OspA and OspC also have a role in protecting the spirochaetes from innate defence molecules in the incoming blood. Both OspC and OspA can bind plasminogen ${ }^{54}$ and promote conversion to plasmin, a protease that negatively regulates the complement system, thus facilitating movement through tick and host tissues ${ }^{55}$. OspA has also been shown to shield spirochaetes from antibodies in the tick gut ${ }^{56}$ and is regulated by several complex global gene expression pathways that facilitate the transition back and forth between tick and vertebrate hosts. One of these pathways is the RpoN-RpoS alternative sigma factor cascade, in which RpoS functions as a gatekeeper to repress tick-phase genes, including ospA, during the mammalian host phase $\mathrm{e}^{52}$. Following spirochaete acquisition by ticks, RpoS is downregulated and a second pathway, the Hk1-Rrp1 two-component system, is activated. During the tick-borne phase of the B. burgdorferi life cycle, Hk1-Rrp1 controls a broad subset of genes that promote survival of spirochaetes in the gut through synthesis of the second messenger c-di-GMP, which in turn induces the expression of several outer surface proteins that inhibit complement-mediated lysis ${ }^{57-62}$. When either the sensor kinase $(\mathrm{Hk} 1)$ or the diguanylate cyclase response regulator (Rrp1) is rendered non-functional, spirochaetes retain the ability to infect mice but are unable to survive acquisition by ticks ${ }^{58,59,62}$. It was reported that while Hk1-Rrp1-deficient spirochaetes were ingested and visible in the guts of ticks feeding on infected mice, the spirochaetes were later eliminated from the gut during the course of feeding. These same mutants were able to persist in the guts of ticks into which they had been injected via a needle; however, once the ticks were allowed to feed on mice, the spirochaete burden also declined, suggesting that the mutants were unable to survive mammalian host factors within the bloodmeal ${ }^{58-60,62}$. Later, during reciprocal tick-to-host transmission, synthesis of RpoS induces a set of genes that facilitate physiological adaptation to the bloodmeal, chemotactic migration out of the 


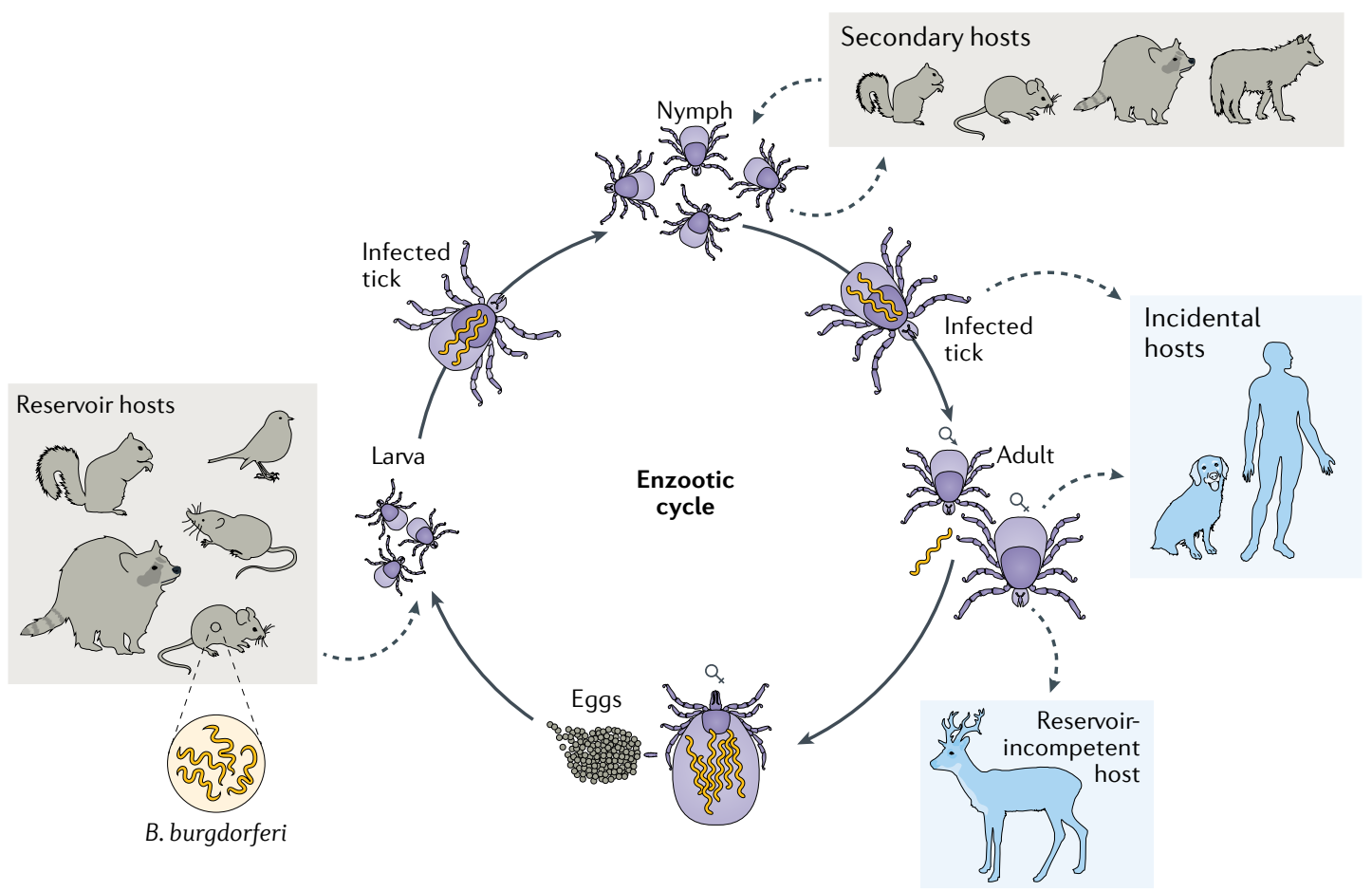

Fig. 1 | The life cycles of Ixodes scapularis and Borrelia burgdorferi. Uninfected larvae hatch and seek a host to feed on, which is typically a small mammal or bird, but may include larger animals. Because Borrelia burgdorferi is not transmitted transovarially, this life stage is the primary opportunity for spirochaetes to infect ticks that feed on an infected host. After feeding, the six-legged larvae moult and emerge as eight-legged nymphs, which may be infected with spirochaetes acquired during their initial bloodmeal. Nymphs seek a second host, typically a small or medium-sized mammal, and this bloodmeal may offer a second opportunity for spirochaetes to infect ticks. Importantly, nymphs infected during the larval bloodmeal can transmit spirochaetes to hosts, including humans and domestic animals. After fed nymphs have moulted to the adult stage, newly emerged adult lxodes scapularis ticks search for a large animal host, typically whitetailed deer, for mating and a final bloodmeal. Although deer are the preferred hosts, adult female ticks will also feed on humans and domestic animals, which can acquire B. burgdorferi, but are relatively unimportant to further perpetuation of infections. Because ticks cannot acquire B. burgdorferi from deer, these hosts are not effective reservoirs for B. burgdorferi, although they are important for perpetuation of tick populations. After mating, engorged females release themselves from hosts and eventually oviposit an egg mass, which may contain hundreds to thousands of eggs. I. scapularis ticks produce only a single clutch of eggs and then die. Solid arrows denote progression steps in the tick life cycle and dashed arrows denote host preferences for specific tick life stages.

Borrelial lymphocytoma A skin lesion that presents as a blue-red skin nodule characterized by robust infiltration of B lymphocytes following Borrelia spp. infection.

\section{Phenology}

The study of how climate and seasons can impact the life cycle of a population. gut and transition to a form that is infectious for mammalian hosts ${ }^{51,59,63-66}$. In addition, BadR (Borrelia host adaptation regulator) represses the transcription of $r p o S$, whereas BosR activates $r p o S$ transcription and represses osp $A$ and $o s p B^{67-70}$. BadR-deficient spirochaetes cannot infect mice, presumably because they lack the ability to repress RpoS and lack the subsequent ability to transition from expression of tick-phase genes to mammalian phase genes ${ }^{69,70}$.

BBE31 is another outer surface lipoprotein with a recognized role in Borrelia-vector interactions. Peak expression of BBE31 occurs in the gut of nymphs on days 2 and 3 after feeding, suggesting an effective role during the period when transmission of spirochaetes occurs ${ }^{71}$. Antibody fragments to BBE31 introduced during this period did not affect $B$. burgdorferi burden in the gut, yet reduced spirochaete presence in haemolymph and salivary glands, presumably though interference with migration out of the gut. TRE31 is a secreted tick protein and a specific binding partner of BBE31. B. burgdorferi infection induces TRE31 expression; it is expressed in the gut of fed and unfed I. scapularis but not in the salivary glands. Blocking expression of TRE31 in feeding ticks had no effect on the presence of spirochaetes in the gut; however, there was a clear reduction of the bacterial burdens in haemolymph and salivary glands, indicating that BBE31-TRE31 interaction is important for transmission to new hosts ${ }^{71}$.

Another important factor in the vector stage of $B$. burgdorferi is the outer membrane surface protein BBA52, which is upregulated early during tick feeding ${ }^{72}$. A mutant lacking functional bba52 was able to establish infection in needle-inoculated mice; however, tick-to-mouse transmission of spirochaetes was inhibited, and tick acquisition of mutants from hosts was also reduced $^{72}$. Although the specific function of this gene is not well understood, it is clearly essential to the entry of spirochaetes to and their exit from the tick.

Recently, a yeast surface display library encoding tick gut proteins was used to identify interactions between whole B. burgdorferi spirochaetes and tick gut proteins. In this study, the authors identified two proteins, Ixofin3D and ISDLP, as potential B. burgdorferi interactants ${ }^{73}$. Ixofin3D is a fibronectin III domain-containing gut 

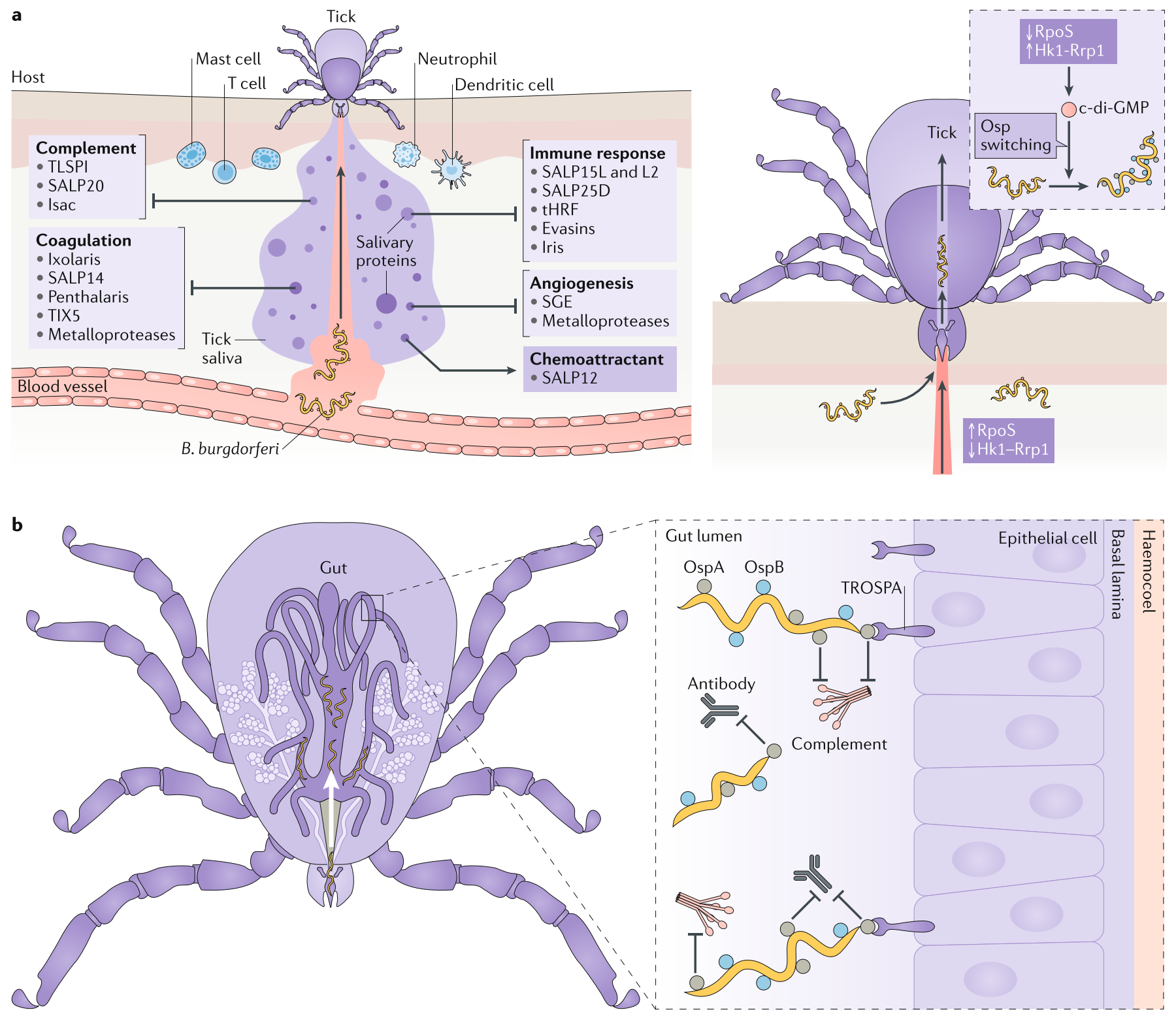

Fig. 2 | Acquisition of Borrelia burgdorferi. a | When feeding on an infected host, the lxodes scapularis tick takes up Borrelia burgdorferi with the bloodmeal. The tick injects the salivary protein SALP25D into the host skin to dampen inflammation at the feeding site. SALP25D quenches reactive oxygen species generated by activated neutrophils at the bite site ${ }^{186}$. The activity of SALP25D enhances B. burgdorferi acquisition by the tick, possibly by increasing viability of the spirochaetes at the bite site. SALP12 functions as a chemoattractant for B. burgdorferi and increases the quantity of spirochetes entering the tick ${ }^{36}$. Entry of B. burgdorferi into the tick induces upregulation of Hk1-Rrp1 and downregulation of RpoS, resulting in production of c-di-GMP, an important molecule regulating expression of outer surface proteins. b | Spirochaetes ingested in the bloodmeal adhere to the tick gut and remain in this organ until a subsequent tick feeding. B. burgdorferi expresses the outer surface proteins OspA and OspB, which protect spirochaetes from harmful components in host blood ${ }^{56}$, including antibodies and complement, and enable them to adhere to and persist in the gut ${ }^{44,45,47}$. OspA interacts specifically with the tick receptor TROSPA, which is located on the luminal surface of gut epithelial cells and is upregulated when spirochaetes are ingested ${ }^{48}$. tHRF, tick histamine release factor.

protein and binds $B$. burgdorferi, facilitating its congregation towards epithelial cells. Although the B. burgdorferi genome encodes at least two proteins that bind host fibronectin, BBK32 (REF. ${ }^{74}$ ) and RevA ${ }^{75}$, they are associated with infection in mice ${ }^{76,77}$ but they are not essential in the tick phase ${ }^{78}$, and neither protein bound Ixofin3D. Therefore, the specific mechanism of interaction of this protein with $B$. burgdorferi remains unclear; however from a functional perspective, it was suggested that the aforementioned congregation would help direct the spirochaete away from the gut lumen and promote its exit from the gut $^{73}$. ISDLP is a dystroglycan-like tick protein and is expressed abundantly on the surface of gut epithelial cells. It bound to B. burgdorferi and facilitated spirochaete migration from the gut by mechanisms that also remain to be determined ${ }^{79}$. 
Similarly to many zoonotic pathogens, $B$. burgdorferi lacks certain metabolic pathways and depends on its hosts for essential nutrients ${ }^{4}$. Because I. scapularis feeds only once per life stage, during much of the tick lifespan, available nutrients are limited, and B. burgdorferi must compete with the tick and its microbiota for nutrients. However, to assist with this, B. burgdorferi uses a transport system to scavenge nutrients from its hosts ${ }^{80,81}$ and maintain metabolic flexibility to use the different nutrients available in arthropod and vertebrate environments. This includes acquisition of carbon, where during infection of mammals, glucose is the primary source of carbon in blood ${ }^{82}$, whereas glycerol and, to a lesser extent, chitobiose are available to spirochaetes in the tick environment ${ }^{62,83-85}$. This was demonstrated by the observation that $B$. burgdorferi mutants lacking a gene necessary for glycerol metabolism could infect mice yet demonstrated a reduced capacity to replicate in ticks ${ }^{84}$.

Influence of the tick microbiota. All multicellular eukaryotes coexist with a collection of commensal microorganisms primarily in the gut ${ }^{86,87}$. This mutualistic partnership provides important benefits for overall gut health, immune responses, pathogen sensing and metabolic functions ${ }^{88}$. Although most microbiota research has been performed in vertebrates, the importance of the microbiota in arthropods has become an exciting topic recently ${ }^{89,90}$. Similarly to the microbiota of other metazoans, the tick microbiota is likely composed of bacteria, archaea, fungi and viruses ${ }^{91}$, but bacteria are the predominant members. Several recent studies and reviews have characterized microbiota diversity in ticks ${ }^{34,91-97}$. There are differing opinions on the extent of the diversity of the tick microbiota. Although several studies have suggested that hundreds of bacterial genera are associated with Ixodes ticks, it is becoming apparent that the abundance and diversity of the tick microbiota may be inflated by spurious contamination due potentially to the low biomass of tick samples ${ }^{34}$. There is growing evidence that the tick microbiota is likely less complex than initially thought ${ }^{34,98}$, composed predominantly of the endosymbiont Rickettsia buchneri $i^{99}$ and other bacterial genera, such as Enterococcus, Pseudomonas, Staphylococcus, Lysinibacillus and Bacillus, at much lower abundance ${ }^{31,33,34,98}$. The rickettsial endosymbiont of Ixodes pacificus has been suggested to provide folate, a key vitamin absent in the bloodmeal, to the tick ${ }^{100}$. Thus, it is likely that rickettsial endosymbionts are preferred and vital inhabitants of the tick. Although the tick microbiota might be unstable, with the exception of $R$. buchneri, the bacterial members that associate with the tick, even if transiently, may impact the tick and its interactions with the pathogens it harbours. Differences in findings are in part due to the development of technologies and approaches to study the microbiota, in addition to technical issues, such as geography, sex, temperature, stage of development and wild versus laboratory-grown ticks ${ }^{31,32,91,93,101-107}$. Here we focus on several studies that have associated microbiota composition in ticks with susceptibility to B. burgdorferi infection ${ }^{31,33,108}$.

A study in 2014 demonstrated that the microbiota has an important role in facilitating B. burgdorferi colonization $^{32}$. In this study, the authors produced dysbiosed I. scapularis larvae by placing surface-sterilized female progenitors in sterile containers, thus limiting the exposure of eggs and hatching larval ticks to the normal environmental microbiota. Larval ticks raised in sterile containers harboured decreased relative abundance of Acinetobacter spp., Brevibacterium spp., Lysinibacillus spp. and Staphylococcus spp. compared with ticks grown in normal laboratory conditions. Dysbiosis resulted in decreased B. burgdorferi colonization when the larvae were allowed to feed on infected mice, suggesting that the microbiota impedes effective B. burgdorferi colonization $^{32}$. While the state of the microbiota can influence colonization efficiency, B. burgdorferi can also actively alter the microbiota during the course of infection to generate an environment that is conducive for colonization ${ }^{33}$. When I. scapularis ticks fed on B. burgdorferi-infected mice, spirochaete presence increased gene expression of several tick genes in the gut such as the gene encoding PIXR (protein of I. scapularis with a reeler domain). PIXR inhibits biofilm formation, and RNA interference-mediated knockdown of PIXR decreased B. burgdorferi colonization, suggesting that $B$. burgdorferi induces PIXR expression to enhance colonization in the tick ${ }^{33}$. Changes to biofilm formation could impact the ability of B. burgdorferi to adhere to the gut epithelium and traverse intercellular junctions in order to access the haemocoel and migrate to the salivary glands ${ }^{41}$.

The microbiota could impact B. burgdorferi colonization through various mechanisms. The genome of B. burgdorferi lacks several genes important for different metabolic pathways, such as synthesis of nucleotides, fatty acids and thiamin ${ }^{4,28,109}$; therefore, spirochaetes rely on the arthropod host and the bloodmeal to acquire these essential nutrients and metabolic products. Endosymbionts have an important nutritional role for arthropods ${ }^{110}$; therefore, alterations to the microbiota could impact B. burgdorferi infection. A study queried the genomes of several Borrelia species, including B. burgdorferi and Borrelia afzelii, and showed that Borrelia spp. lack interbacterial effector and immunity genes that would be crucial for survival in a polymicrobial milieu $^{34}$. Consistent with this rationale, the abundance of Pesudomonas, Bacillus or Enterobacteriaceae was negatively correlated with $B$. burgdorferi abundance ${ }^{34,91}$. Additional studies are required for a mechanistic understanding of the impact of the microbiota on B. burgdorferi survival in the tick.

Alterations to the tick microbiota also disrupt the structural integrity of the peritrophic matrix, which provides a barrier between the incoming bloodmeal and the gut epithelium $^{32,111}$. Changes to the microbiota decreased expression of peritrophin, a key structural component, and disruption of the peritrophic membrane, which reduced $B$. burgdorferi colonization and adherence to the gut lumen ${ }^{32}$. These alterations to the microbiota were accompanied by decreased immune activation through the JAK-STAT pathway ${ }^{32}$ (FIG. 3). Demonstrating a functional link between STAT and the integrity of the peritrophic membrane, the study showed that STAT was a transcriptional activator of several members of 
the peritrophin family of genes on the basis of the presence of canonical STAT-binding sites in their promoter regions ${ }^{32}$. Differential STAT activation could also potentially impact the tick immune responses towards the spirochaetes, as discussed in the next subsection ${ }^{32}$.

The tick immune system. Ticks, like arthropods, have an immune system to detect and control potential pathogens. Therefore, $B$. burgdorferi has evolved strategies to evade tick immune defences. Most of our knowledge of the arthropod immune system comes from research in

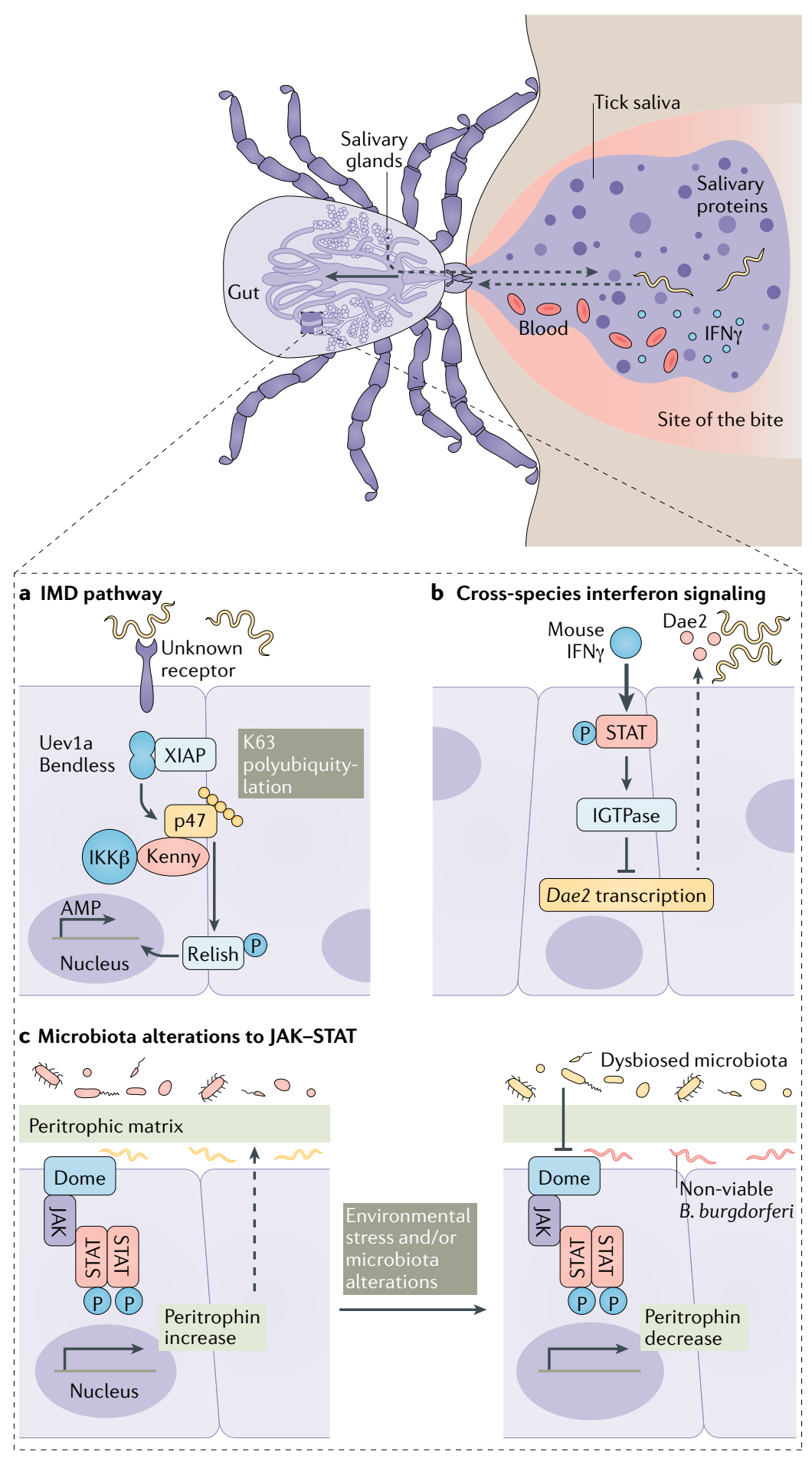

Drosophila melanogaster ${ }^{12,113}$. The arthropod immune system consists of the immune deficiency (IMD) pathway, the JAK-STAT pathway and Toll receptor signalling ${ }^{113-117}$. Activation of these pathways can occur through various mechanisms, including recognition of pathogen-associated molecular patterns and activation of the Toll receptor ligand Spaetzle, or induction of cytokine-like molecules ${ }^{118}$. In Drosophila, activation of these pathways triggers the production of several effector molecules, including antimicrobial peptides, which contributes to controlling infection by invading bacteria, viruses or fungi ${ }^{118}$. Unlike Drosophila, in which specific antimicrobial peptides are activated by specific immune pathways, relatively limited information is available in ticks. Several recent computational studies have characterized the immune system in ticks and identified key similarities and differences with Drosophila ${ }^{25,26,119}$. Initial studies to characterize the I. scapularis immune system identified 234 genes that were categorized into nine immune pathways: gut-microorganism homeostasis, agglutination, leucine-rich repeat proteins, proteases, coagulation, non-self recognition and transduction via Toll, IMD pathway and JAK-STAT pathways, free radical defence, phagocytosis and antimicrobial peptides ${ }^{119}$. The recent sequencing of the $I$. scapularis genome expanded on these findings and identified additional components encoded in the IMD, JAK-STAT and Toll signalling pathways ${ }^{21}$. Despite missing several components of the immune signalling pathways, ticks maintain a functional immune response against invading pathogens $^{22,26,30,120-122}$.

The IMD pathway in Drosophila has been well characterized and is primarily important for resistance to Gram-negative bacteria ${ }^{118,123-125}$. The IMD pathway is activated by diaminopimelic peptidoglycans binding

\section{Fig. 3 | Tick immune defences against Borrelia} burgdorferi infection. a Infection with Borrelia burgdorferi activates the immune deficiency (IMD) pathway in Ixodes scapularis. On activation, $\mathrm{p} 47$ is polyubiquitylated by $X$-linked inhibitor of apoptosis (XIAP) in complex with the Bendless-Uev1a heterodimer. Downstream signalling through Kenny results in phosphorylation of Relish and transcription of antimicrobial peptides (AMPs). b | Uninfected I. scapularis can ingest mouse interferon- $\gamma($ IFN $\gamma)$ along with the bloodmeal when feeding on B. burgdorferi infected mice. Mouse IFN $\gamma$ signals in the tick gut through an unknown receptor, resulting in STAT-mediated activation of a Rholike GTPase (IGTPase) and production of the AMP Dae2. c|Certain microbiota compositions enable activation of the JAK-STAT pathway by an unknown molecular signal and in turn induce the expression of peritrophin genes. Peritrophins are crucial for the formation of a structurally intact peritrophic matrix. B. burgdorferi uses the peritrophic matrix as a shield or barrier protecting it from toxic contents during colonization of the gut epithelium. STAT-induced immune effectors, as well as environmental changes, can alter the microbiota composition. Certain changes in the microbiota composition impair JAK-STAT signalling, and this results in decreased expression of peritrophins, leading to a thinner and compromised peritrophic matrix. A compromised peritrophic matrix no longer functions as a protective shield and thus impairs B. burgdorferi colonization of the gut epithelium. 
to peptidoglycan recognition proteins, resulting in the production of antimicrobial peptides ${ }^{112,116}$. I. scapularis encodes several genes involved in IMD pathway activation, such as caudal, relish, tak1, posh and caspar; however, several signalling components are absent ${ }^{21,25,26}$. Although ticks are missing various proteins involved in the IMD pathway signalling cascade, several important proteins have been identified that are involved in the activation of the IMD pathway on infection with B. burgdorferi, as well as A. phagocytophilum ${ }^{21,22,25,29}$. On infection and pathogen detection, the tick E3 ubiquitin ligase $\mathrm{X}$-linked inhibitor of apoptosis (XIAP) ubiquitylates p47, allowing p47 to activate the NF- $\mathrm{KB}$ regulator Kenny ${ }^{29}$ (FIG. 3). This interaction between XIAP and p47 is critical for IMD pathway activation and nuclear translocation of the NF- $\kappa \mathrm{B}$ homologue Relish, which is important for antimicrobial peptide expression ${ }^{29,126}$. B. burgdorferi does not contain diaminopimelic peptidoglycans in its envelope ${ }^{127}$. Instead, lipids that make up the bacterial membrane, 1-palmitoyl-2-oleoyl-sn-glycero-3-phosphoglycerol and 1-palmitoyl-2-oleoyl diacylglycerol, activate the IMD pathway ${ }^{22}$. Additionally, RNAi knockdown of several tick genes involved in IMD signalling, such as caspar, relish, uev1a and bendless, resulted in increased B. burgdorferi burden in ticks allowed to feed on infected mice $^{22}$. Overall, these results clearly demonstrate activation of the IMD pathway on infection with B. burgdorferi; however, additional studies to identify the effector antimicrobial peptides involved in killing and/or inhibiting B. burgdorferi are still needed.

Several recent studies have identified a role for the JAK-STAT pathway in controlling B. burgdorferi infection in ticks. The JAK-STAT pathway is conserved between Drosophila and ticks ${ }^{128}$, and I. scapularis maintains several components of the JAK-STAT pathway, such as stat, hop, dome, pias and socs $^{21}$. I. scapularis does not encode the canonical ligand Upd, a secreted protein in Drosophila, which binds and activates the JAK receptor Dome ${ }^{21,129}$. Nevertheless, despite lacking $u p d$, ticks maintain a functional JAK-STAT pathway and use this pathway to control pathogen burden ${ }^{30,121}$. A recent study identified cross-species cytokine signalling between mice and ticks ${ }^{30}$. In this study, when I. scapularis ticks were allowed to feed on $B$. burgdorferi-infected mice, interferon- $\gamma(\mathrm{IFN} \gamma)$ was also ingested with the bloodmeal. Murine-derived IFN $\gamma$ activated I. scapularis STAT in the gut through an unknown receptor ${ }^{30}$. Activated STAT induced activation of a tick-encoded Rho-like GTPase (IGTPase) and production of the antimicrobial peptide Dae2 (FIG. 3). Dae2 is suggested to be an important antimicrobial peptide for controlling B. burgdorferi during acquisition, although a direct effect of Dae2 on B. burgdorferi remains to be demonstrated ${ }^{122}$. Knockdown of Dae2 in I. scapularis resulted in increased B. burgdorferi burden after feeding on infected mice. Importantly, activation of the JAK-STAT pathway has also been observed on infection with A. phagocytophilum ${ }^{121}$. A. phagocytophilum infection activated the JAK-STAT pathway, resulting in expression of a $5.3-\mathrm{kDa}$ antimicrobial peptide. RNAi knockdown of STAT increased A. phagocytophilum burden in salivary glands and increased transmission ${ }^{121}$.
The Toll signalling pathway has been well studied in Drosophila and has an important role in defence against Gram-positive bacteria ${ }^{118,130,131}$. Activation of the Toll pathway occurs through the Toll receptor ligand Spaetzle, which is secreted in an inactive form and cleaved on extracellular detection of bacterial components, such as lysine-type peptidoglycan. Spaetzle binding activates the Toll receptor, resulting in binding to the adaptor protein MyD88 and downstream signalling ${ }^{132}$. I. scapularis encodes several components of the Toll signalling cascade, such as toll, myd88, spaetzle, tube, pelle, cactus and dorsal ${ }^{21}$. Additionally, expression of several toll genes and myd88 is upregulated on infection with B. burgdorferi ${ }^{30}$. However, the role of Toll activation in controlling B. burgdorferi infection has not been determined and is an important area of future research.

The previously described studies demonstrate that the JAK-STAT and IMD pathways have important roles in controlling B. burgdorferi infection ${ }^{22,29,30,122}$. However, although B. burgdorferi infection activates the JAKSTAT and IMD pathways, these defence systems are not able to eliminate the spirochaete from the tick. Little is known regarding how $B$. burgdorferi can circumvent restriction by the IMD and JAK-STAT pathways in the tick gut.

The B. burgdorferi genome encodes several proteins to control immune activation in mammals during transmission, such as BBA57, which dampens interferon activation $^{133}$. Whether the $B$. burgdorferi genome encodes proteins that impair tick immune activation pathways through direct protein-protein interactions is unknown and requires further investigation. A possible mechanism to dampen activation of tick immune defences could be through the formation of a molecular barrier that surrounds the gut epithelial layer, termed the 'dityrosine network' (DTN) ${ }^{134}$. In mosquitoes, formation of the DTN limits immune activation during a bloodmeal, which could potentially disrupt the beneficial gut microbiota ${ }^{134}$. Similarly to other arthropods, I. scapularis encodes a dual oxidase (duox) and a peroxidase (ISCW017368) involved in DTN formation ${ }^{135}$. Knockdown of duox or the peroxidase impaired DTN formation, resulting in activation of tick immunity and reduced B. burgdorferi colonization ${ }^{135}$. Silencing of duox also increased nitric oxide synthase activity. Production of reactive nitrogen species is an additional protective mechanisms that ticks use to control pathogens, such as B. burgdorferi, by targeting DNA $^{136,137}$. However, B. burgdorferi can counter the nitrosative stress through expression of the nucleotide excision repair gene $u v r B^{137}$. Alternatively, as B. burgdorferi infection persists through multiple life stages of the tick, $B$. burgdorferi has evolved strategies to minimize fitness costs to the tick. It is possible that I. scapularis becomes tolerant of B. burgdorferi infection because immune activation could be detrimental to the host.

\section{Interactions in the salivary gland}

Once at the salivary gland, $B$. burgdorferi is transmitted with the tick saliva during feeding. Tick feeding is a dynamic process that involves penetrating the epidermis, digesting tissue, dilating capillaries, preventing coagulation and dampening immune responses at the 
a

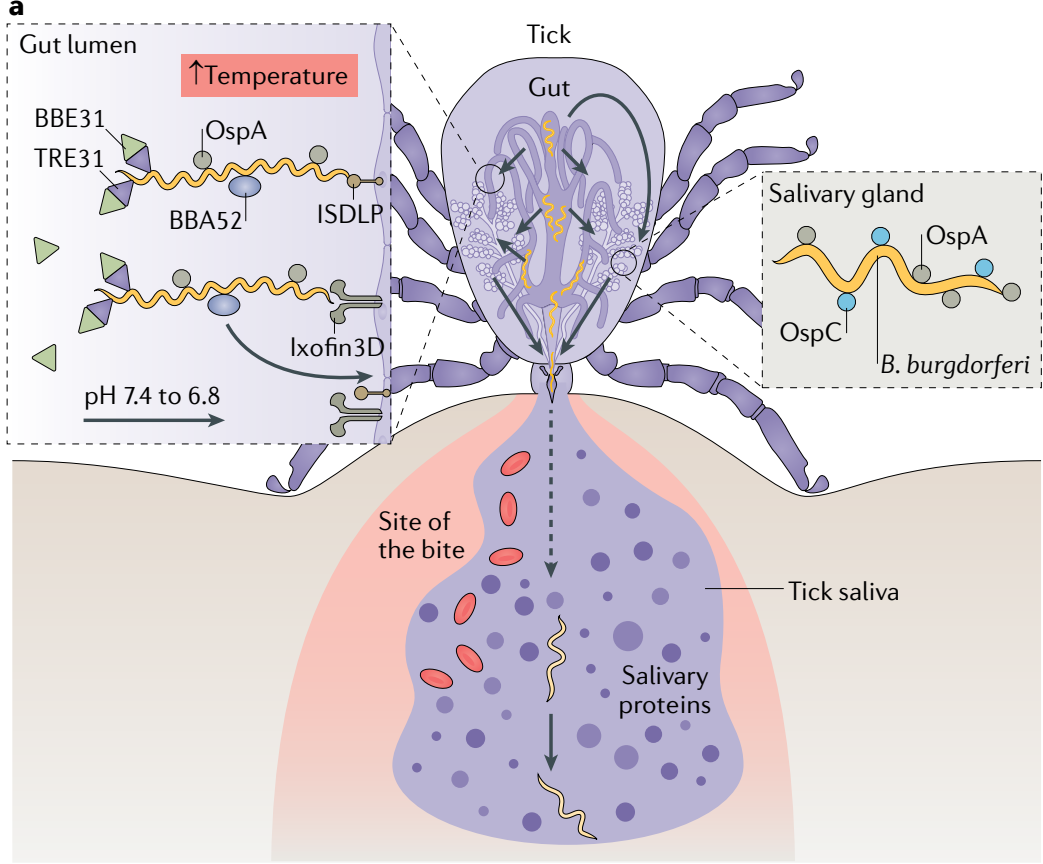

b
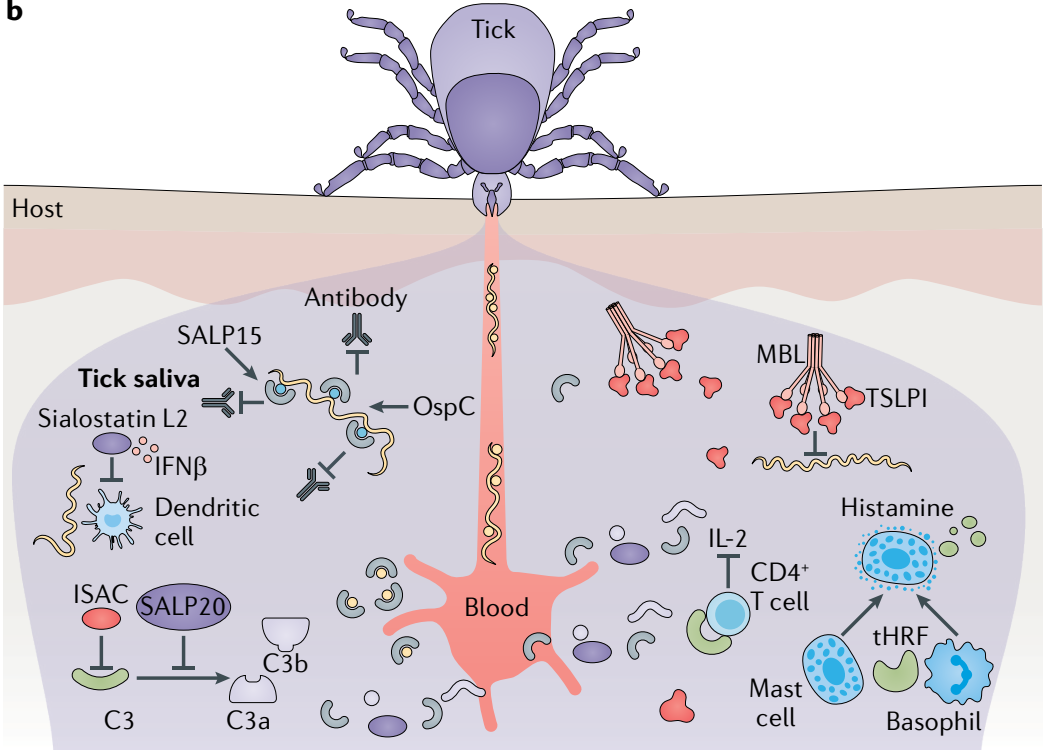

Type $2 \mathrm{~T}$ helper cell response Immune response by effector $\mathrm{CD}^{+} \mathrm{T}$ cells characterized by production of cytokines promoting B cell proliferation and antibody production.

Type $1 \mathrm{~T}$ helper cell response Immune response by effector $\mathrm{CD}^{+}{ }^{+} T$ cells characterized by production of proinflammatory cytokines involved in the killing intracellular pathogens.

Hypostome

A component of a tick's mouthpart apparatus that serves to anchor the tick in the host's skin during feeding. bite site ${ }^{138}$. These processes are mediated by proteins injected into the host through the tick saliva ${ }^{139}$. Tick saliva contains at least several hundred proteins, possibly thousands ${ }^{24}$. Saliva composition varies between life stages of the tick, the type of host it is feeding on and also during feeding to adapt to the changing conditions encountered during feeding ${ }^{23,140-142}$. For example, the tick secretes an adhesive cement-like substance shortly after insertion of the mouthparts to anchor itself in the skin. Subsequently, saliva composition shifts to proteins that facilitate acquisition of the bloodmeal and immune evasion, and eventually wound healing and detachment from the host $\mathrm{t}^{23,143,144}$. One study found that the composition of I. scapularis saliva changed every 24 hours, although it is likely that salivary proteome changes occur at shorter intervals ${ }^{23}$. Although the primary function of these proteins is to facilitate acquisition of a bloodmeal,
Fig. 4 | Transmission of Borrelia burgdorferi to a vertebrate host. a | Several environmental changes that occur at the onset of tick feeding are cues for spirochaetes in the gut to transition to a form that is infectious for vertebrates and to initiate migration to the salivary glands. Outer surface proteins important for this process include BBA52, which is upregulated during the early stages of feeding $^{72}$, and BBE31, which interacts with the tick receptor TRE31 to enable the spirochaetes to exit the gut epithelial layer and migrate through the haemocoel to the salivary glands ${ }^{71}$. Ixofin3D and ISDLP are other proteins expressed by epithelial cells that bind spirochaetes and are thought to assist in exit from the gut ${ }^{32,79}$. Spirochaetes outside the gut express OspA and OspC, which promotes binding to tick salivary glands and early dissemination in the vertebrate host $^{10,197} \cdot \mathbf{b}$ | Transmission of Borrelia burgdorferi to a mammalian host is enhanced by the activity of several tick salivary proteins. As the tick feeds, several proteins are secreted into the host to modulate the host environment and to obtain a complete bloodmeal. These proteins also assist $B$. burgdorferi transmission. Complement is an important immune defence mechanism that restricts B. burgdorferi, as well as tick feeding. The tick salivary proteins ISAC, SALP20 and TSLPI inhibit activation of complement and increase B. burgdorferi transmission ${ }^{164,179,180}$. Sialostatin L2 also modulates the immune response against the tick bite by impairing cytokine secretion by dendritic cells on exposure to $B$. burgdorferi ${ }^{167}$. Tick histamine release factor (tHRF) is a salivary protein secreted during the late stage of tick feeding and triggers the release of histamine, presumably from mast cells or basophils ${ }^{185}$. The best studied salivary protein is SALP15, which enhances B. burgdorferi transmission. B. burgdorferi expresses OspC on its surface during migration from the gut to the salivary glands. SALP15 binds OspC and can shield the spirochaete from antibody-mediated killing ${ }^{190}$. Additionally, SALP15 suppresses $\mathrm{CD} 4^{+} \mathrm{T}$ cell function and IL-2 secretion ${ }^{161}$.

they can also benefit B. burgdorferi transmission ${ }^{145}$ (FIG. 4). For example, the immune response against $B$. burgdorferi is highly dependent on tick salivary proteins. Mice infected with B. burgdorferi developed a type $2 \mathrm{~T}$ helper cell response, whereas mice inoculated with a syringe developed a mixed type $2 \mathrm{~T}$ helper cell and type $1 \mathrm{~T}$ helper cell response $\mathrm{e}^{146}$. These differences are likely mediated, at least in part, by the proteins in saliva. Saliva and salivary gland extract can downregulate IFN $\gamma$ and IL-2 production in T cells stimulated with concanavalin $\mathrm{A}$ and inhibit $\mathrm{T}$ cell proliferation ${ }^{147,148}$. By contrast, B. burgdorferi alone induces IFN $\gamma$ production ${ }^{149}$. In the following subsections, we will discuss key salivary proteins that facilitate a successful bloodmeal and how they impact B. burgdorferi transmission and/or acquisition.

Salivary proteins important for tick feeding. Innate defences in the skin are a major hurdle for ticks to overcome to obtain a complete bloodmeal. In short, ticks digest tissue, insert their hypostome and take in blood, which pools at the wound ${ }^{138}$. Injury to skin normally triggers wound healing, which consists of three overlapping phases - inflammation, tissue formation and tissue repair ${ }^{144}$. Wound healing could be detrimental to tick feeding; therefore, ticks have evolved strategies to modulate host response. Initial damage to the skin disrupts blood vessels, resulting in release of extracellular 
adenosine triphosphate and adenosine diphosphate, which stimulates platelet aggregation and inhibits blood flow $^{150}$. Ticks use several strategies to maintain blood flow. The saliva of Ixodes spp. ticks contains an apyrase enzyme that degrades adenosine diphosphate to adenosine monophosphate and thus inhibits platelet aggregation ${ }^{151}$. Similarly, saliva proteins in I. scapularis such as SALP14, TIX, Ixolaris, and Penthalaris have anticoagulation properties ${ }^{152-154}$. Knockdown of SALP14 by RNAi reduced the anticoagulation activity of saliva, as measured by the activity of complement factor Xa, which is important for thrombin activation. SALP14 knockdown also inhibited tick feeding and reduced engorgement by $50-70 \%$ (REF. ${ }^{152}$ ). Several additional proteins, such as ISL 929, and metalloproteinases also have important roles in controlling wound healing in response to the tick bite bin-158. $^{150}$.

Skin contains several resident immune cells, such as macrophages, mast cells, dendritic cells, Langerhans cells and T cells, that are important for protection against invading pathogens ${ }^{159}$. Evasion of the immune response is crucial for the tick as feeding involves injection of foreign proteins into the host over the course of several days. Ticks have evolved several strategies to control recruitment of inflammatory cells and cells involved in tissue repair. Tick saliva and salivary gland extract inhibit angiogenesis at the bite site ${ }^{157,160}$. Additionally, saliva dampens the inflammatory immune response by directly targeting immune cells and complement activation at the tick bite site ${ }^{161-164}$. Several proteins in saliva inhibit $\mathrm{T}$ cell proliferation and decrease type $1 \mathrm{~T}$ helper cell cytokine production $^{165,166}$. For example, sialostatin L2 suppresses the immune response by interfering with activation of the JAK-STAT pathway and IFN $\beta$ production in murine dendritic cells ${ }^{167}$. In addition to controlling inflammation at the bite site, sialostatin L2 has an important role during pathogen transmission ${ }^{168,169}$. Sialostatin L2 inhibits the formation of the NLRC4 inflammasome during transmission of $A$. phagocytophilum by binding annexin A2, which is required for NLRC4 oligomerization and inflammasome formation ${ }^{168,170}$. Immunization of guinea pigs against sialostatin L2 resulted in decreased engorgement, suggesting that the immunosuppressive function of sialostatin L2 is important for tick feeding ${ }^{171}$.

Salivary proteins that facilitate Borrelia transmission. The complement system is an important component of the innate immune system involved in clearance of invading pathogens. There are three major pathways, lectin, classical and alternative, that can activate complement ${ }^{172}$. On activation, effector pathways result in opsonization and phagocytosis, generation of proinflammatory anaphylatoxins or direct lysis of the pathogen by the formation of membrane attack complexes ${ }^{172}$. Complement activation has an important role in controlling B. burgdorferi dissemination in mice ${ }^{173,174}$; therefore, $B$. burgdorferi has evolved mechanisms to counter the effects of the complement cascade through expression of complement inhibitor proteins on the outer membrane, such as complement regulatory-acquiring surface proteins (CRASPS), which directly interfere with complement activation ${ }^{175-177}$. Several studies have reported that tick salivary proteins can be important in controlling complement activation to enhance B. burgdorferi transmission ${ }^{163,164}$. Multiple salivary proteins from I. scapularis can inhibit the complement pathway, such as Isac, TSLPI and SALP20 (REFS ${ }^{163,164,178,179}$ ). For example, SALP20 from I. scapularis inhibits the alternative complement pathway by preventing cleavage of $\mathrm{C} 3$ to $\mathrm{C} 3 \mathrm{a}$ and $\mathrm{C} \mathrm{b}^{178}$. Furthermore, SALP20 partially protected B. burgdorferi from complement mediated lysis ${ }^{180}$.

Knockdown of TSLPI in ticks impaired B. burgdorferi transmission to mice ${ }^{164}$. Similarly, infected ticks initiated less pathogen transmission to mice immunized against TSLPI than non-immunized mice. In vitro, B. burgdorferi was resistant to complement-mediated killing in the presence of recombinant TSLPI. TSLPI binds mannose-binding lectin, a pattern recognition protein involved in activation of the lectin pathway, and thereby TLSPI inhibits the lectin-complement cascade ${ }^{164,181}$. Additionally, mannose-binding lectin is involved in controlling B. burgdorferi infection in the $\operatorname{skin}^{182}$.

Controlling the host's immune response during the different phases of tick feeding is critical for obtaining a successful bloodmeal. Histamine released from degranulated basophils and mast cells has a negative impact on tick feeding within the first 24 hours from attachment ${ }^{183,184}$. Therefore, ticks secrete several histamine-binding proteins to reduce the detrimental effects of histamine on feeding and attachment; however, ticks also encode proteins to stimulate degranulation during the late phase of tick feeding ${ }^{185}$. Tick histamine release factor (tHRF) is upregulated in the salivary glands of infected I. scapularis nymphs during the late stages of feeding ${ }^{185}$. tHRF stimulates the release of histamine from basophils in mice, possibly as a mechanism to increase blood flow and pooling. Knockdown of tHRF impaired tick feeding on mice, and mice that received passive transfer of tHRF antiserum also showed decreased B. burgdorferi transmission.

Neutrophils are the first cells to be recruited to the site of acute inflammation. SALP25D is a secreted salivary protein important for combating neutrophils at the bite site ${ }^{186,187}$. Recombinant SALP25D protected spirochaetes from activated neutrophils compared with control protein. Knockdown of SALP25D in I. scapularis impaired B. burgdorferi acquisition in ticks ${ }^{187}$. Additionally, the study authors demonstrated that SALP25D is an antioxidant protein that quenches reactive oxygen species generated by activated neutrophils. Although the $B$. burgdorferi genome encodes enzymes that remove reactive oxygen species, SALP25D is a clear example of $B$. burgdorferi co-opting a vector protein to help maintain its life cycle ${ }^{186}$. Furthermore, two secreted salivary proteins, ISL929 and ISL1373, which belong to a family of disintegrin-like proteins, reduced neutrophil recruitment in vivo ${ }^{158}$, and this function provided a substantial advantage for B. burgdorferi at the vector-host interface, representing yet another example of saliva-assisted transmission ${ }^{188}$. SALP15 is a unique example of a salivary protein that promotes $B$. burgdorferi transmission by binding to the spirochaete. SALP15 also binds to $\mathrm{CD}^{+} \mathrm{T}$ cells and inhibits $\mathrm{T}$ cell activation and IL-2 signalling ${ }^{161,189}$. SALP15 interacts directly with 
B. burgdorferi during migration out of the tick, and is able to enhance transmission to naive mice ${ }^{190}$. Mice inoculated with spirochaetes preincubated with recombinant SALP15 had a higher B. burgdorferi burden compared with mice preincubated with a control protein. SALP15 binds directly to OspC on the spirochaetes, protecting them from antibody-mediated killing ${ }^{163,190}$. Knockdown of SALP15 in ticks strongly reduced transmission to mice. Mice actively immunized with SALP15 were also partially protected against infection with tick-borne B. burgdorferi ${ }^{191}$.

\section{Conclusions}

B. burgdorferi has evolved complex mechanisms to infect both vertebrates and arthropods. An expanding set of research tools is facilitating an increasingly deeper understanding of these features. The recent sequencing of the I. scapularis genome and bioinformatics work has improved our understanding of Borrelia-tick interactions $s^{21,25,26}$. These studies have helped to expand our understanding of the tick innate immune defence pathways and interactions with $B$. burgdorferi. This work has also provided a useful resource for comparative genomics and arthropod evolutionary biology. Additionally, development of tick cell lines has led to major advances in our ability to study tick-pathogen interactions for I. scapularis in vitro ${ }^{192-194}$, including isolation of tick-borne pathogens, expression of RNA and proteins, genetic manipulation and knockout studies.
As described herein, B. burgdorferi relies on interactions with the tick for successful colonization, persistence and transmission. With the recent advancements in our understanding of the tick immune system and microbiota, further analysis is required to identify how gene expression changes in B. burgdorferi help to modulate the tick environment. Several recent studies clearly demonstrate that the tick IMD and JAK-STAT pathways have roles in controlling infection. However, further studies are required to determine how B. burgdorferi can avoid clearance and identify the proteins involved. Additionally, studies to restore components of the JAKSTAT, IMD and Toll signalling pathways absent in ticks would be an interesting research direction to explore. Similarly, studies are required to examine how the tick microbiota can impact metabolic functions of the tick and B. burgdorferi. Further characterization of these interactions, as well as interactions at the tick bite site, could help to identify potentially druggable targets to disrupt infection and transmission. For example, salivary proteins enhance transmission of $B$. burgdorferi and $A$. phagocytophilum to the vertebrate host. Targeting these interactions in the salivary glands has become an exciting approach for vaccine development ${ }^{195,196}$. Developing immunity against tick salivary proteins can be a strategy to prevent tick bites, as well as infection with the pathogens they transmit.

Published online 10 July 2020
1. Hinckley, A. F. et al. Lyme disease testing by large commercial laboratories in the United States. Clin. Infect. Dis. 59, 676-681 (2014).

2. Rosenberg, R. et al. Vital signs: trends in reported vectorborne disease cases - United States and territories, 2004-2016. MMWR 67, 496-501 (2018).

3. Hyde, J. A. Borrelia burgdorferi keeps moving and carries on: a review of borrelial dissemination and invasion. Front. Immunol. 8, 114 (2017).

4. Fraser, C. M. et al. Genomic sequence of a Lyme disease spirochaete, Borrelia burgdorferi. Nature 390, 580-586 (1997).

5. Ferdows, M. S. \& Barbour, A. G. Megabase-sized linear DNA in the bacterium Borrelia burgdorferi, the Lyme disease agent. Proc. Natl Acad. Sci. USA 86, 5969-5973 (1989).

6. Samuels, D. S. Gene regulation in Borrelia burgdorferi. Annu. Rev. Microbiol. 65, 479-499 (2011).

7. Carroll, J. A., Garon, C. F. \& Schwan, T. G. Effects of environmental $\mathrm{pH}$ on membrane proteins in Borrelia burgdorferi. Infect. Immun. 67, 3181-3187 (1999).

8. Hyde, J. A., Trzeciakowski, J. P. \& Skare, J. T. Borrelia burgdorferi alters its gene expression and antigenic profile in response to $\mathrm{CO}_{2}$ levels. J. Bacteriol. 189 , 437-445 (2007)

9. Tokarz, R., Anderton, J. M., Katona, L. I. \& Benach, J. L. Combined effects of blood and temperature shift on Borrelia burgdorferi gene expression as determined by whole genome DNA array. Infect. Immun. 72 5419-5432 (2004).

10. Schwan, T. G., Piesman, J., Golde, W. T., Dolan, M. C. $\&$ Rosa, P. A. Induction of an outer surface protein on Borrelia burgdorferi during tick feeding. Proc. Natl Acad. Sci. USA 92, 2909-2913 (1995).

11. LoGiudice, K., Ostfeld, R. S., Schmidt, K. A. \& Keesing, F. The ecology of infectious disease: effects of host diversity and community composition on Lyme disease risk. Proc. Natl Acad. Sci. USA 100, 567-571 (2003).

12. Piesman, J., Spielman, A., Etkind, P., Ruebush, T. K. II \& Juranek, D. D. Role of deer in the epizootiology of Babesia microti in Massachusetts, USA. J. Med. Entomol. 15, 537-540 (1979).

13. Gatewood, A. G. et al. Climate and tick seasonality are predictors of Borrelia burgdorferi genotype distribution. Appl. Env. Microbiol. 75, 2476-2483 (2009).
14. Ogden, N. H. et al. Evidence for geographic variation in life-cycle processes affecting phenology of the Lyme disease vector Ixodes scapularis (Acari: Ixodidae) in the United States. J. Med. Entomol. 55, 1386-1401 (2018).

15. Stromdahl, E. et al. Comparison of phenology and pathogen prevalence, including infection with the Ehrlichia muris-like (EML) agent, of Ixodes scapularis removed from soldiers in the midwestern and the northeastern United States over a 15 year period (1997-2012). Parasit. Vectors 7, 553 (2014).

16. Hamer, S. A. et al. Wild birds and urban ecology of ticks and tick-borne pathogens, Chicago, Illinois, USA, 2005-2010. Emerg. Infect. Dis. 18, 1589-1595 (2012).

17. Spielman, A., Wilson, M. L., Levine, J. F. \& Piesman, J. Ecology of Ixodes dammini-borne human babesiosis and Lyme disease. Annu. Rev. Entomol. 30, 439-460 (1985).

18. Piesman, J. Transmission of Lyme disease spirochetes (Borrelia burgdorferi). Exp. Appl. Acarol. 7, 71-80 (1989).

19. Mead, P. S. Epidemiology of Lyme disease. Infect. Dis. Clin. North. Am. 29, 187-210 (2015)

20. Eisen, R. J., Kugeler, K. J., Eisen, L., Beard, C. B. \& Paddock, C. D. Tick-borne zoonoses in the United States: persistent and emerging threats to human health. ILAR J. 58, 319-335 (2017).

21. Gulia-Nuss, M. et al. Genomic insights into the Ixodes scapularis tick vector of Lyme disease. Nat. Commun. 7, 10507 (2016).

22. Shaw, D. K. et al. Infection-derived lipids elicit an immune deficiency circuit in arthropods. Nat. Commun. 8, 14401 (2017)

23. Kim, T. K. et al. Ixodes scapularis tick saliva proteins sequentially secreted every $24 \mathrm{~h}$ during blood feeding. PLoS Negl. Trop. Dis. 10, e0004323 (2016).

24. Perner, J., Kropackova, S., Kopacek, P. \& Ribeiro, J. M. C Sialome diversity of ticks revealed by RNAseq of single tick salivary glands. PLoS Negl. Trop. Dis. 12, e0006410 (2018).

25. Bechsgaard, J. et al. Comparative genomic study of arachnid immune systems indicates loss of beta1,3-glucanase-related proteins and the immune deficiency pathway. J. Evol. Biol. 29, 277-291 (2016).
26. Rosa, R. D et al. Exploring the immune signalling pathway-related genes of the cattle tick Rhipicephalus microplus: from molecular characterization to transcriptional profile upon microbial challenge. Dev. Comp. Immunol. 59, 1-14 (2016)

27. Phelan, J. P. et al. Genome-wide screen identifies nove genes required for Borrelia burgdorferi survival in its Ixodes tick vector. PLoS Pathog. 15, e1007644 (2019).

28. Zhang, K. et al. Lyme disease spirochaete Borrelia burgdorferi does not require thiamin. Nat. Microbiol. 2, 16213 (2016)

29. McClure Carroll, E. E. et al. p47 licenses activation of the immune deficiency pathway in the tick Ixodes scapularis. Proc. Natl Acad. Sci. USA 116, 205-210 (2019).

30. Smith, A. A. et al. Cross-species interferon signaling boosts microbicidal activity within the tick vector. Cell Host Microbe 20, 91-98 (2016).

31. Abraham, N. M. et al. Pathogen-mediated manipulation of arthropod microbiota to promote infection. Proc. Natl Acad. Sci. USA 114, E781-E790 (2017).

32. Narasimhan, S. et al. Gut microbiota of the tick vector Ixodes scapularis modulate colonization of the Lyme disease spirochete. Cell Host Microbe 15, 58-71 (2014).

33. Narasimhan, S. et al. Modulation of the tick gut milieu by a secreted tick protein favors Borrelia burgdorferi colonization. Nat. Commun. 8, 184 (2017).

34. Ross, B. D et al. Ixodes scapularis does not harbor a stable midgut microbiome. ISME J. 12, 2596-2607 (2018).

35. Nakamura, S. Spirochete flagella and motility. Biomolecules 10, 550 (2020).

36. Murfin, K. E., Kleinbard, R., Aydin, M., Salazar, S. A \& Fikrig, E. Borrelia burgdorferi chemotaxis toward tick protein Salp1 2 contributes to acquisition. Ticks Tick Borne Dis. 10, 1124-1134 (2019).

37. Zung, J. L. et al. Fine structural evidence for the penetration of the Lyme disease spirochete Borrelia burgdorferi through the gut and salivary tissues of Ixodes dammini. Can. J. Zool. 67, 1737-1748 (1989).

38. Franta, Z. et al. Dynamics of digestive proteolytic system during blood feeding of the hard tick Ixodes ricinus. Parasit. Vectors 3, 119 (2010). 
39. De Silva, A. M. \& Fikrig, E. Growth and migration of Borrelia burgdorferi in Ixodes ticks during blood feeding. Am. J. Trop. Med. Hyg. 53, 397-404 (1995).

40. de la Fuente, J., Estrada-Pena, A., Venzal, J. M. Kocan, K. M. \& Sonenshine, D. E. Overview: ticks as vectors of pathogens that cause disease in humans and animals. Front. Biosci. 13, 6938-6946 (2008).

41. Dunham-Ems, S. M. et al. Live imaging reveals a biphasic mode of dissemination of Borrelia burgdorfer within ticks. J. Clin. Invest. 119, 3652-3665 (2009).

42. Ramamoorthy, R. \& Scholl-Meeker, D. Borrelia burgdorferi proteins whose expression is similarly affected by culture temperature and $\mathrm{pH}$. Infect. Immun. 69, 2739-2742 (2001).

43. Yang, X. et al. Interdependence of environmental factors influencing reciprocal patterns of gene expression in virulent Borrelia burgdorferi. $\mathrm{Mol}$. Microbiol. 37, 1470-1479 (2000).

44. Pal, U. et al. Attachment of Borrelia burgdorferi within Ixodes scapularis mediated by outer surface protein $A$ J. Clin. Invest. 106, 561-569 (2000).

45. Fikrig, E., Pal, U., Chen, M., Anderson, J. F. \& Flavell, R. A. OspB antibody prevents Borrelia burgdorferi colonization of Ixodes scapularis. Infect. Immun. 72, 1755-1759 (2004).

46. Yang, X. F., Pal, U., Alani, S. M., Fikrig, E. \& Norgard, M. V. Essential role for OspA/B in the life cycle of the Lyme disease spirochete. J. Exp. Med. 199, 641-648 (2004).

47. Neelakanta, G. et al. Outer surface protein B is critical for Borrelia burgdorferi adherence and survival within Ixodes ticks. PLoS Pathog. 3, e33 (2007)

48. Pal, U. et al. TROSPA, an Ixodes scapularis receptor for Borrelia burgdorferi. Cell 119, 457-468 (2004)

49. Pal, U. et al. OspC facilitates Borrelia burgdorferi invasion of Ixodes scapularis salivary glands. J. Clin. Invest. 113, 220-230 (2004).

50. de Silva, A. M., Telford, S. R. III, Brunet, L. R. Barthold, S. W. \& Fikrig, E. Borrelia burgdorferi OspA is an arthropod-specific transmission-blocking Lyme disease vaccine. J. Exp. Med. 183, 271-275 (1996).

51. Dunham-Ems, S. M., Caimano, M. J., Eggers, C. H. \& Radolf, J. D. Borrelia burgdorferi requires the alternative sigma factor RpoS for dissemination within the vector during tick-to-mammal transmission. PLOS Pathog. 8, e1002532 (2012)

52. Caimano, M. J. et al. The RpoS gatekeeper in Borrelia burgdorferi: an invariant regulatory scheme that promotes spirochete persistence in reservoir hosts and niche diversity. Front. Microbiol. 10, 1923 (2019).

53. Ohnishi, J., Piesman, J. \& de Silva, A. M. Antigenic and genetic heterogeneity of Borrelia burgdorferi populations transmitted by ticks. Proc. Natl Acad Sci. USA 98, 670-675 (2001).

54. Fuchs, H., Wallich, R., Simon, M. M. \& Kramer, M. D. The outer surface protein A of the spirochete Borrelia burgdorferi is a plasmin(ogen) receptor. Proc. Natl Acad. Sci. USA 91, 12594-12598 (1994).

55. Coleman, J. L. et al. Borrelia burgdorferi binds plasminogen, resulting in enhanced penetration of endothelial monolayers. Infect. Immun. 63 2478-2484 (1995).

56. Bunikis, J. \& Barbour, A. G. Access of antibody or trypsin to an integral outer membrane protein (P66) of Borrelia burgdorferi is hindered by Osp lipoproteins. Infect. Immun. 67, 2874-2883 (1999).

57. Rogers, E. A. et al. Rrp1, a cyclic-di-GMP-producing response regulator, is an important regulator of Borrelia burgdorferi core cellular functions. Mol. Microbiol. 71, 1551-1573 (2009).

58. Caimano, M. J. et al. The hybrid histidine kinase Hk 1 is part of a two-component system that is essential for survival of Borrelia burgdorferi in feeding Ixodes scapularis ticks. Infect. Immun. 79, 3117-3130 (2011)

59. Kostick, J. L. et al. The diguanylate cyclase, Rrp1, regulates critical steps in the enzootic cycle of the Lyme disease spirochetes. Mol. Microbiol. 81 219-231 (2011)

60. Caimano, M. J. et al. Cyclic di-GMP modulates gene expression in Lyme disease spirochetes at the tickmammal interface to promote spirochete survival during the blood meal and tick-to-mammal transmission. Infect. Immun. 83, 3043-3060 (2015).

61. Kraiczy, P. \& Stevenson, B. Complement regulator acquiring surface proteins of Borrelia burgdorferi: structure, function and regulation of gene expression. Ticks Tick Borne Dis. 4, 26-34 (2013).

62. He, M. et al. Cyclic di-GMP is essential for the survival of the Lyme disease spirochete in ticks. PLoS Pathog. 7, e1002133 (2011).
63. Tilly, K. et al. Borrelia burgdorferi OspC protein required exclusively in a crucial early stage of mammalian infection. Infect. Immun. 74, 3554-3564 (2006).

64. Weening, E. H. et al. Borrelia burgdorferi lacking DbpBA exhibits an early survival defect during experimental infection. Infect. Immun. $\mathbf{7 6}$ 5694-5705 (2008).

65. Eggers, C. H. et al. The coenzyme A disulphide reductase of Borrelia burgdorferi is important for rapid growth throughout the enzootic cycle and essential for infection of the mammalian host. Mol. Microbiol. 82, 679-697 (2011).

66. Fisher, M. A. et al. Borrelia burgdorferi sigma54 is required for mammalian infection and vector transmission but not for tick colonization. Proc. Natl Acad Sci. USA 102, 5162-5167 (2005).

67. Ouyang, Z., Deka, R. K. \& Norgard, M. V. BosR (BB0647) controls the RpoN-RpoS regulatory pathway and virulence expression in Borrelia burgdorferi by a novel DNA-binding mechanism. PLoS Pathog. 7 e1001272 (2011).

68. Ouyang, Z., Zhou, J. \& Norgard, M. V. Synthesis of RpoS is dependent on a putative enhancer binding protein Rrp2 in Borrelia burgdorferi. PLOS ONE 9 , e96917 (2014).

69. Ouyang, Z. \& Zhou, J. BadR (BB0693) controls growth phase-dependent induction of rpoS and bosR in Borrelia burgdorferi via recognizing TAAAATAT motifs. Mol. Microbiol. 98, 1147-1167 (2015)

70. Miller, C. L., Karna, S. L. \& Seshu, J. Borrelia host adaptation regulator (BadR) regulates rpoS to modulate host adaptation and virulence factors in Borrelia burgdorferi. Mol. Microbiol. 88, 105-124 (2013).

71. Zhang, L. et al. Molecular interactions that enable movement of the Lyme disease agent from the tick gut into the hemolymph. PLoS Pathog. 7, e1002079 (2011).

72. Kumar, M., Yang, X., Coleman, A. S. \& Pal, U. BBA52 facilitates Borrelia burgdorferi transmission from feeding ticks to murine hosts. J. Infect. Dis. 201 , 1084-1095 (2010)

73. Narasimhan, S. et al. A tick gut protein with fibronectin III domains aids Borrelia burgdorfer congregation to the gut during transmission. PLoS Pathog. 10, e1004278 (2014).

74. Probert, W. S. \& Johnson, B. J. Identification of a $47 \mathrm{kDa}$ fibronectin-binding protein expressed by Borrelia burgdorferi isolate B31. Mol. Microbiol. 30, 1003-1015 (1998)

75. Brissette, C. A., Bykowski, T., Cooley, A. E., Bowman, A $\&$ Stevenson, B. Borrelia burgdorferi RevA antigen binds host fibronectin. Infect. Immun. 77, 2802-2812 (2009).

76. Seshu, J. et al. Inactivation of the fibronectin-binding adhesin gene bbk32 significantly attenuates the infectivity potential of Borrelia burgdorferi. $\mathrm{Mol}$. Microbiol. 59, 1591-1601 (2006).

77. Byram, R. et al. Borrelia burgdorferi RevA significantly affects pathogenicity and host response in the mouse model of Lyme disease. Infect. Immun. $\mathbf{8 3}$ 3675-3683 (2015)

78. Li, X. et al. The Lyme disease agent Borrelia burgdorferi requires BB0690, a Dps homologue, to persist within ticks. Mol. Microbiol. 63, 694-710 (2007).

79. Coumou, J. et al. Ixodes scapularis dystroglycan-like protein promotes Borrelia burgdorferi migration from the gut. J. Mol. Med. 94, 361-370 (2016)

80. Kung, F., Anguita, J. \& Pal, U. Borrelia burgdorferi and tick proteins supporting pathogen persistence in the vector. Future Microbiol. 8, 41-56 (2013).

81. Caimano, M. J., Drecktrah, D., Kung, F. \& Samuels, D. S. Interaction of the Lyme disease spirochete with its tick vector. Cell Microbiol. 18, 919-927 (2016).

82. Hoon-Hanks, L. L. et al. Borrelia burgdorferi malO mutants utilize disaccharides and traverse the enzootic cycle. FEMS Immunol. Med. Microbiol. 66, 157-165 (2012).

83. Tilly, K., Grimm, D., Bueschel, D. M., Krum, J. G. \& Rosa, P. Infectious cycle analysis of a Borrelia burgdorferi mutant defective in transport of chitobiose, a tick cuticle component. Vector Borne Zoonotic Dis. 4, 159-168 (2004).

84. Pappas, C. J. et al. Borrelia burgdorferi requires glycerol for maximum fitness during the tick phase of the enzootic cycle. PLoS Pathog. 7, e1002102 (2011).

85. Sze, C. W. et al. Study of the response regulator Rrp1 reveals its regulatory role in chitobiose utilization and virulence of Borrelia burgdorferi. Infect. Immun. $\mathbf{8 1}$, 1775-1787 (2013).
86. Ley, R. E. et al. Evolution of mammals and their gut microbes. Science 320, 1647-1651 (2008).

87. Levy, M., Kolodziejczyk, A. A., Thaiss, C. A. ¿ Elinav, E. Dysbiosis and the immune system. Nat. Rev. Immunol. 17, 219-232 (2017)

88. Clemente, J. C., Ursell, L. K., Parfrey, L. W. \& Knight, R. The impact of the gut microbiota on human health: an integrative view. Cell 148, 1258-1270 (2012).

89. Shin, S. C. et al. Drosophila microbiome modulates host developmental and metabolic homeostasis via insulin signaling. Science 334, 670-674 (2011).

90. Boissiere, A. et al. Midgut microbiota of the malaria mosquito vector Anopheles gambiae and interactions with Plasmodium falciparum infection. PLoS Pathog. 8, e1002742 (2012)

91. Landesman, W. J., Mulder, K., Fredericks, L. P. \& Allan, B. F. Cross-kingdom analysis of nymphal-stage xodes scapularis microbial communities in relation to Borrelia burgdorferi infection and load. FEMS Microbiol. Ecol. 95 (2019).

92. Van Treuren, W. et al. Variation in the microbiota of xodes ticks with regard to geography, species, and sex. Appl. Env. Microbiol. 81, 6200-6209 (2015).

93. Narasimhan, S. \& Fikrig, E. Tick microbiome: the force within. Trends Parasitol. 31, 315-323 (2015).

94. Diaz-Sanchez, S., Estrada-Pena, A., Cabezas-Cruz, A. $\&$ de la Fuente, J. Evolutionary insights into the tick hologenome. Trends Parasitol. 35, 725-737 (2019).

95. Swei, A. \& Kwan, J. Y. Tick microbiome and pathogen acquisition altered by host blood meal. ISME J. 11 , 813-816 (2017).

96. Kwan, J. Y., Griggs, R., Chicana, B., Miller, C. \& Swei, A. Vertical vs. horizontal transmission of the microbiome in a key disease vector, Ixodes pacificus. Mol. Ecol. 26 6578-6589 (2017).

97. Rynkiewicz, E. C., Hemmerich, C., Rusch, D. B. Fuqua, C.\& Clay, K. Concordance of bacterial communities of two tick species and blood of their shared rodent host. Mol. Ecol. 24, 2566-2579 (2015).

98. Couper, L. \& Swei, A. Tick microbiome characterization by next-generation $16 \mathrm{~S}$ rRNA amplicon sequencing. J. Vis. Exp. https://doi.org/10.3791/58239 (2018).

99. Kurtti, T. J. et al. Rickettsia buchneri sp. nov., a rickettsial endosymbiont of the blacklegged tick Ixodes scapularis. Int. J. Syst. Evol. Microbiol. 65, 965-970 (2015)

100. Hunter D. J et al. The Rickettsia endosymbiont of Ixodes pacificus contains all the genes of de novo folate biosynthesis. PLOS ONE 10, e0144552 (2015).

101. Moreno, C. X., Moy, F., Daniels, T. J., Godfrey, H. P. $\&$ Cabello, F. C. Molecular analysis of microbial communities identified in different developmental stages of Ixodes scapularis ticks from Westchester and Dutchess counties, New York. Env. Microbiol. 8 761-772 (2006)

102. Benson, M. J., Gawronski, J. D., Eveleigh, D. E. \& Benson, D. R. Intracellular symbionts and other bacteria associated with deer ticks (Ixodes scapularis) from Nantucket and Wellfleet, Cape Cod, Massachusetts. Appl. Env. Microbiol. 70, 616-620 (2004).

103. Landesman, W. J et al. Potential effects of blood meal host on bacterial community composition in Ixodes scapularis nymphs. Ticks Tick Borne Dis. 10, 523-527 (2019).

104. Thapa, S., Zhang, Y. \& Allen, M. S. Bacterial microbiomes of Ixodes scapularis ticks collected from Massachusetts and Texas, USA. BMC Microbiol. 19, 138 (2019).

105. Tokarz, R. et al. Microbiome analysis of Ixodes scapularis ticks from New York and Connecticut. Ticks Tick Borne Dis. 10, 894-900 (2019).

106. Thapa, S., Zhang, Y. \& Allen, M. S. Effects of temperature on bacterial microbiome composition in Ixodes scapularis ticks. Microbiologyopen 8, e00719 (2019).

107. Obregon, D., Bard, E., Abrial, D., Estrada-Pena, A. $\Sigma$ Cabezas-Cruz, A. Sex-specific linkages between taxonomic and functional profiles of tick gut microbiomes. Front. Cell Infect. Microbiol. 9, 298 (2019).

108. Gall, C. A. et al. The bacterial microbiome of Dermacentor andersoni ticks influences pathogen susceptibility. ISME J. 10, 1846-1855 (2016).

109. Corona, A. \& Schwartz, I. Borrelia burgdorferi: carbon metabolism and the tick-mammal enzootic cycle. Microbiol. Spectr. 3 (2015).

110. Bonnet, S. I., Binetruy, F., Hernandez-Jarguin, A. M $\&$ Duron, O. The tick microbiome: why non-pathogenic microorganisms matter in tick biology and pathogen transmission. Front. Cell Infect. Microbiol. 7, 236 (2017). 
111. Hegedus, D., Erlandson, M., Gillott, C. \& Toprak, U. New insights into peritrophic matrix synthesis, architecture, and function. Annu. Rev. Entomol. 54, 285-302 (2009)

112. Lemaitre, B. \& Hoffmann, J. The host defense of Drosophila melanogaster. Annu. Rev. Immunol. 25, 697-743 (2007).

113. Hoffmann, J. A., Kafatos, F. C., Janeway, C. A. \& Ezekowitz, R. A. Phylogenetic perspectives in innate immunity. Science 284, 1313-1318 (1999).

114. Xi, Z., Ramirez, J. L. \& Dimopoulos, G. The Aedes aegypti toll pathway controls dengue virus infection. PLoS Pathog. 4, e 1000098 (2008)

115. Souza-Neto, J. A., Sim, S. \& Dimopoulos, C. An evolutionary conserved function of the JAK-STAT pathway in anti-dengue defense. Proc. Natl Acad. Sci. USA 106, 17841-17846 (2009).

116. Kaneko, T. et al. Monomeric and polymeric gramnegative peptidoglycan but not purified LPS stimulate the Drosophila IMD pathway. Immunity 20, 637-649 (2004).

117. Dostert, C. et al. The Jak-STAT signaling pathway is required but not sufficient for the antiviral response of drosophila. Nat. Immunol. 6, 946-953 (2005).

118. Hoffmann, J. A. \& Reichhart, J. M. Drosophila innate immunity: an evolutionary perspective. Nat. Immunol. 3, 121-126 (2002)

119. Smith, A. A. \& Pal, U. Immunity-related genes in Ixodes scapularis-perspectives from genome information. Front. Cell Infect. Microbiol. 4, 116 (2014).

120. Sonenshine, D. E., Ceraul, S. M., Hynes, W. E., Macaluso, K. R. \& Azad, A. F. Expression of defensinlike peptides in tick hemolymph and midgut in response to challenge with Borrelia burgdorferi, Escherichia coli and Bacillus subtilis. Exp. Appl. Acarol. 28, 127-134 (2002)

121. Liu, L. et al. Ixodes scapularis JAK-STAT pathway regulates tick antimicrobial peptides, thereby controlling the agent of human granulocytic anaplasmosis. J. Infect. Dis. 206, 1233-1241 (2012).

122. Chou, S. et al. Transferred interbacterial antagonism genes augment eukaryotic innate immune function. Nature 518, 98-101 (2015)

123. Leulier, F., Rodriguez, A., Khush, R. S., Abrams, J. M $\&$ Lemaitre, B. The Drosophila caspase Dredd is required to resist gram-negative bacterial infection. EMBO Rep. 1, 353-358 (2000).

124. Georgel, P. et al. Drosophila immune deficiency (IMD) is a death domain protein that activates antibacterial defense and can promote apoptosis. Dev. Cell 1 503-514 (2001).

125. Lemaitre, B. et al. A recessive mutation, immune deficiency (imd), defines two distinct control pathways in the Drosophila host defense. Proc. Natl Acad. Sci. USA 92, 9465-9469 (1995).

126. Erturk-Hasdemir, D. et al. Two roles for the Drosophila IKK complex in the activation of Relish and the induction of antimicrobial peptide genes. Proc. Natl Acad. Sci. USA 106, 9779-9784 (2009).

127. Beck, G., Benach, J. L. \& Habicht, G. S. Isolation, preliminary chemical characterization, and biological activity of Borrelia burgdorferi peptidoglycan. Biochem. Biophys. Res. Commun. 167, 89-95 (1990).

128. Palmer, W. J. \& Jiggins, F. M. Comparative genomics reveals the origins and diversity of arthropod immune systems. Mol. Biol. Evol. 32, 2111-2129 (2015).

129. Harrison, D. A., McCoon, P. E., Binari, R., Gilman, M. \& Perrimon, N. Drosophila unpaired encodes a secreted protein that activates the JAK signaling pathway. Genes Dev. 12, 3252-3263 (1998).

130. Michel, T., Reichhart, J. M., Hoffmann, J. A. \& Royet, J. Drosophila toll is activated by gram-positive bacteria through a circulating peptidoglycan recognition protein. Nature 414, 756-759 (2001).

131. Rutschmann, S., Kilinc, A. \& Ferrandon, D. Cutting edge: the toll pathway is required for resistance to gram-positive bacterial infections in Drosophila. J. Immunol. 168, 1542-1546 (2002).

132. Valanne, S., Wang, J. H. \& Ramet, M. The Drosophila toll signaling pathway. J. Immunol. 186, 649-656 (2011)

133. Bernard, Q. et al. Plasticity in early immune evasion strategies of a bacterial pathogen. Proc. Natl Acad. Sci. USA 115, E3788-E3797 (2018).

134. Kumar, S., Molina-Cruz, A., Gupta, L., Rodrigues, J. $\&$ Barillas-Mury, C. A peroxidase/dual oxidase system modulates midgut epithelial immunity in Anopheles gambiae. Science 327, 1644-1648 (2010).
135. Yang, X., Smith, A. A., Williams, M. S. \& Pal, U. A dityrosine network mediated by dual oxidase and peroxidase influences the persistence of Lyme disease pathogens within the vector. J. Biol. Chem. 289, 12813-12822 (2014).

136. Bourret, T. J., Boylan, J. A., Lawrence, K. A. \& Gherardini, F. C. Nitrosative damage to free and zinc-bound cysteine thiols underlies nitric oxide toxicity in wild-type Borrelia burgdorferi. Mol. Microbiol. 81, 259-273 (2011).

137. Bourret, T. J. et al. The nucleotide excision repair pathway protects borrelia burgdorferi from nitrosative stress in Ixodes scapularis ticks. Front. Microbiol. 7 1397 (2016)

138. Anderson, J. F. \& Magnarelli, L. A. Biology of ticks. Infect. Dis. Clin. North. Am. 22, 195-215 (2008).

139. Francischetti, I. M., Sa-Nunes, A., Mans, B. J., Santos, I. M. \& Ribeiro, J. M. The role of saliva in tick feeding. Front. Biosci. 14, 2051-2088 (2009).

140. Ribeiro, J. M. et al. An annotated catalog of salivary gland transcripts from Ixodes scapularis ticks. Insect Biochem. Mol. Biol. 36, 111-129 (2006).

141. Valenzuela, J. G. et al. Exploring the sialome of the tick Ixodes scapularis. J. Exp. Biol. 205, 2843-2864 (2002).

142. Narasimhan, S. et al. Host-specific expression of Ixodes scapularis salivary genes. Ticks Tick Borne Dis. 10, 386-397 (2019).

143. Anderson, J. M. et al. Ticks, Ixodes scapularis, feed repeatedly on white-footed mice despite strong inflammatory response: an expanding paradigm for understanding tick-host interactions. Front. Immunol. 8, 1784 (2017).

144. Singer, A. J. \& Clark, R. A. Cutaneous wound healing. N. Engl. J. Med. 341, 738-746 (1999).

145. Zeidner, N. S., Schneider, B. S., Nuncio, M. S., Gern, L. \& Piesman, J. Coinoculation of Borrelia spp. with tick salivary gland lysate enhances spirochete load in mice and is tick species-specific. J. Parasitol. 88, 1276-1278 (2002).

146. Christe, M., Rutti, B. \& Brossard, M. Cytokines (IL-4 and IFN-gamma) and antibodies (IgE and IgG2a) produced in mice infected with Borrelia burgdorferi sensu stricto via nymphs of Ixodes ricinus ticks or syringe inoculations. Parasitol. Res. 86, 491-496 (2000).

147. Ramachandra, R. N. \& Wikel, S. K. Effects of Dermacentor andersoni (Acari: Ixodidae) salivary gland extracts on Bos indicus and B. taurus lymphocytes and macrophages: in vitro cytokine elaboration and lymphocyte blastogenesis. J. Med. Entomol. 32, 338-345 (1995)

148. Urioste, S., Hall, L. R., Telford, S. R. III \& Titus, R. G. Saliva of the Lyme disease vector, Ixodes dammini, blocks cell activation by a nonprostaglandin E2-dependent mechanism. J. Exp. Med. 180 1077-1085 (1994).

149. Ma, Y. et al. Outer surface lipoproteins of Borrelia burgdorferi stimulate nitric oxide production by the cytokine-inducible pathway. Infect. Immun. $\mathbf{6 2}$ 3663-3671 (1994).

150. Gaarder, A., Jonsen, J., Laland, S., Hellem, A \& Owren, P. A. Adenosine diphosphate in red cells as a factor in the adhesiveness of human blood platelets. Nature 192, 531-532 (1961).

151. Ribeiro, J. M., Makoul, G. T., Levine, J., Robinson, D. R \& Spielman, A. Antihemostatic, antiinflammatory, and immunosuppressive properties of the saliva of a tick. Ixodes dammini. J. Exp. Med. 161, 332-344 (1985).

152. Narasimhan, S. et al. Disruption of Ixodes scapularis anticoagulation by using RNA interference. Proc. Nat Acad. Sci. USA 101, 1141-1146 (2004).

153. Schuijt, T. J. et al. Factor Xa activation of factor $\mathrm{V}$ is of paramount importance in initiating the coagulation system: lessons from a tick salivary protein. Circulation 128, 254-266 (2013).

154. Francischetti, I. M., Valenzuela, J. G., Andersen, J. F., Mather, T. N. \& Ribeiro, J. M. Ixolaris, a nove recombinant tissue factor pathway inhibitor (TFPI) from the salivary gland of the tick, Ixodes scapularis: identification of factor $\mathrm{X}$ and factor $\mathrm{Xa}$ as scaffolds for the inhibition of factor VIla/tissue factor complex. Blood 99, 3602-3612 (2002).

155. Francischetti, I. M., Mather, T. N. \& Ribeiro, J. M. Penthalaris, a novel recombinant five-Kunitz tissue factor pathway inhibitor (TFPI) from the salivary gland of the tick vector of Lyme disease, Ixodes scapularis. Thromb. Haemost. 91, 886-898 (2004).

156. Simo, L., Kazimirova, M., Richardson, J. \& Bonnet, S. I. The essential role of tick salivary glands and saliva in tick feeding and pathogen transmission. Front. Cell Infect. Microbiol. 7, 281 (2017).
157. Francischetti, I. M., Mather, T. N. \& Ribeiro, J. M. Tick saliva is a potent inhibitor of endothelial cell proliferation and angiogenesis. Thromb. Haemost. 94 167-174 (2005)

158. Guo, X. et al. Inhibition of neutrophil function by two tick salivary proteins. Infect. Immun. 77, 2320-2329 (2009).

159. Bos, J. D. \& Kapsenberg, M. L. The skin immune system: progress in cutaneous biology. Immunol. Today 14, 75-78 (1993).

160. Hajnicka, V., Vancova-Stibraniova, I., Slovak, M., Kocakova, P. \& Nuttall, P. A. Ixodid tick salivary gland products target host wound healing growth factors. Int. J. Parasitol. 41, 213-223 (2011).

161. Anguita, J. et al. Salp 15, an ixodes scapularis salivary protein, inhibits $\mathrm{CD} 4^{+} \mathrm{T}$ cell activation. Immunity 16, 849-859 (2002).

162. Hovius, J. W. et al. Salp 15 binding to DC-SIGN inhibits cytokine expression by impairing both nucleosome remodeling and mRNA stabilization. PLoS Pathog. 4, e31 (2008).

163. Schuijt, T. J. et al. The tick salivary protein Salp15 inhibits the killing of serum-sensitive Borrelia burgdorferi sensu lato isolates. Infect. Immun. 76 2888-2894 (2008).

164. Schuijt, T. J. et al. A tick mannose-binding lectin inhibitor interferes with the vertebrate complement cascade to enhance transmission of the Lyme disease agent. Cell Host Microbe 10, 136-146 (2011).

165. Gillespie, R. D., Dolan, M. C., Piesman, J. \& Titus, R. G Identification of an IL-2 binding protein in the saliva of the Lyme disease vector tick, Ixodes scapularis. J. Immunol. 166, 4319-4326 (2001).

166. Kovar, L., Kopecky, J. \& Rihova, B. Salivary gland extract from Ixodes ricinus tick modulates the host immune response towards the Th2 cytokine profile. Parasitol. Res. 88, 1066-1072 (2002).

167. Lieskovska, J. et al. Tick salivary cystatin sialostatin L2 suppresses IFN responses in mouse dendritic cells. Parasite Immunol. 37, 70-78 (2015).

168. Wang, X. et al. The tick protein sialostatin L2 binds to annexin A2 and inhibits NLRC4-mediated inflammasome activation. Infect. Immun. 84, 1796-1805 (2016).

169. Kotsyfakis, M., Horka, H., Salat, J. \& Andersen, J. F. The crystal structures of two salivary cystatins from the tick Ixodes scapularis and the effect of these inhibitors on the establishment of Borrelia burgdorferi infection in a murine model. Mol. Microbiol. 77, 456-470 (2010)

170. Chen, G. et al. The tick salivary protein sialostatin L2 inhibits caspase-1-mediated inflammation during Anaplasma phagocytophilum infection. Infect. Immun 82, 2553-2564 (2014)

171. Kotsyfakis, M. et al. Cutting edge: immunity against a "silent" salivary antigen of the Lyme vector Ixodes scapularis impairs its ability to feed. J. Immunol. 181 5209-5212 (2008).

172. Dunkelberger, J. R. \& Song, W. C. Complement and its role in innate and adaptive immune responses. Cell Res. 20, 34-50 (2010).

173. Lawrenz, M. B. et al. Effect of complement component C3 deficiency on experimental Lyme borreliosis in mice. Infect. Immun. 71, 4432-4440 (2003).

174. Kurtenbach, K., Sewell, H. S., Ogden, N. H., Randolph, S. E. \& Nuttall, P. A. Serum complement sensitivity as a key factor in Lyme disease ecology. Infect. Immun. 66, 1248-1251 (1998).

175. Alitalo, A. et al. Complement evasion by Borrelia burgdorferi: serum-resistant strains promote $\mathrm{C} 3 \mathrm{~b}$ inactivation. Infect. Immun. 69, 3685-3691 (2001).

176. Kraiczy, P. Hide and seek: how lyme disease spirochetes overcome complement attack. Front. Immunol. 7, 385 (2016).

177. Hart, T. et al. Polymorphic factor H-binding activity of CspA protects Lyme borreliae from the host complement in feeding ticks to facilitate tick-to-host transmission. PLoS Pathog 14 e1007106 (2018).

178. Tyson, K. R., Elkins, C. \& de Silva, A. M. A novel mechanism of complement inhibition unmasked by a tick salivary protein that binds to properdin J. Immunol. 180, 3964-3968 (2008).

179. Valenzuela, J. G., Charlab, R., Mather, T. N. \& Ribeiro, J. M. Purification, cloning, and expression of a novel salivary anticomplement protein from the tick, Ixodes scapularis. J. Biol. Chem. 275, 18717-18723 (2000).

180. Tyson, K., Elkins, C., Patterson, H., Fikrig, E. \& de Silva, A. Biochemical and functional characterization of Salp20, an Ixodes scapularis tick salivary protein that inhibits the complement pathway. Insect Mol. Biol. 16, 469-479 (2007) 
181. Ip, W. K., Takahashi, K., Ezekowitz, R. A. \& Stuart, L. M. Mannose-binding lectin and innate immunity. Immunol. Rev. 230, 9-21 (2009).

182. Coumou, J. et al. The role of mannose binding lectin in the immune response against Borrelia burgdorfer sensu lato. Sci. Rep. 9, 1431 (2019).

183. Kemp, D. H. \& Bourne, A. Boophilus microplus: the effect of histamine on the attachment of cattle-tick larvae-studies in vivo and in vitro. Parasitology $\mathbf{8 0}$, 487-496 (1980).

184. Tabakawa, Y. et al. Histamine released from skininfiltrating basophils but not mast cells is crucial for acquired tick resistance in mice. Front. Immunol. 9 , 1540 (2018)

185. Dai, J. et al. Tick histamine release factor is critical for Ixodes scapularis engorgement and transmission of the Lyme disease agent. PLoS Pathog. 6, e1001205 (2010).

186. Das, S. et al. Salp25D, an Ixodes scapularis antioxidant, is 1 of 14 immunodominant antigens in engorged tick salivary glands. J. Infect. Dis. 184 , 1056-1064 (2001).

187. Narasimhan, S. et al. A tick antioxidant facilitates the Lyme disease agent's successful migration from the mammalian host to the arthropod vector. Cell Host Microbe 2, 7-18 (2007).

188. Nuttall, P. A \& Labuda, M. Tick-host interactions: saliva-activated transmission. Parasitology 129 S177-S189 (2004)

189. Garg, R. et al. Cutting edge: CD4 is the receptor for the tick saliva immunosuppressor, Salp15. J. Immunol. 177, 6579-6583 (2006).

190. Ramamoorthi, N. et al. The Lyme disease agent exploits a tick protein to infect the mammalian host. Nature 436, 573-577 (2005).

191. Dai, J. et al. Antibodies against a tick protein, Salp 15 protect mice from the Lyme disease agent. Cell Host Microbe 6, 482-492 (2009)

192. Munderloh, U. G., Liu, Y., Wang, M., Chen, C. \& Kurtti, T. J. Establishment, maintenance and description of cell lines from the tick Ixodes scapularis J. Parasitol. 80, 533-543 (1994).

193. Kurtti, T. J., Munderloh, U. G., Andreadis, T. G., Magnarelli, L. A. \& Mather, T. N. Tick cell culture isolation of an intracellular prokaryote from the tick Ixodes scapularis. J. Invertebr. Pathol. 67, 318-321 (1996).
194. Oliver, J. D., Chavez, A. S., Felsheim, R. F., Kurtti, T. J. \& Munderloh, U. G. An Ixodes scapularis cell line with a predominantly neuron-like phenotype. Exp. Appl. Acarol. 66, 427-442 (2015).

195. Narasimhan, S. et al. Ixodes scapularis saliva components that elicit responses associated with acquired tick-resistance. Ticks Tick Borne Dis. 11, 101369 (2020).

196. Bensaci, M., Bhattacharya, D., Clark, R. \& Hu, L. T. Oral vaccination with vaccinia virus expressing the tick antigen subolesin inhibits tick feeding and transmission of Borrelia burgdorferi. Vaccine 30, 6040-6046 (2012).

197. Grimm, D. et al. Experimental assessment of the roles of linear plasmids Ip25 and Ip28-1 of Borrelia burgdorferi throughout the infectious cycle. Infect. Immun. 72, 5938-5946 (2004).

198. Stanek, G. Wormser, G. P. Gray, J \& Strle, F. Lyme borreliosis. Lancet 379, 461-473 (2012).

199. Pritt, B. S. et al. Borrelia mayonii sp. nov., a member of the Borrelia burgdorferi sensu lato complex, detected in patients and ticks in the upper midwestern United States. Int. J. Syst. Evol. Microbiol. 66, 4878-4880 (2016).

200. Stanek, G. et al. Lyme borreliosis: clinical case definitions for diagnosis and management in Europe. Clin. Microbiol. Infect. 17, 69-79 (2011).

201. Margos, G. et al. Borrelia bavariensis sp. nov. is widely distributed in Europe and Asia. Int. J. Syst. Evol. Microbiol. 63, 4284-4288 (2013).

202. Centers for Disease Control and Prevention. Lyme disease data tables: most recent year (2018). https:// www.cdc.gov/lyme/datasurveillance/tables-recent.html (2018).

203. Lindgren, E., T. G. T. Jaenson. \& Menne, B. Lyme Borreliosis in Europe : influences of climate and climate change, epidemiology, ecology and adaptation measures https://apps.who.int/iris/bitstream/ handle/10665/107800/E89522.pdf?sequence= 1 \&isAllowed=y (WHO, 2006)

204. Barbour, A. G. \& Zückert, W. R. New tricks of tickbourne pathogen. Nature 390, 553-554 (1997)

205. Goldstein, S. F., Charon, N. W. \& Kreiling, J. A. Borrelia burgdorferi swims with a planar waveform similar to that of eukaryotic flagella. Proc. Natl Acad. Sci. USA 91, 3433-3437 (1994)

206. Takayama, K., Rothenberg, R. J. \& Barbour, A. G. Absence of lipopolysaccharide in the Lyme disease spirochete, Borrelia burgdorferi. Infect. Immun. 55, 2311-2313 (1987)

207. Charon, N. W. et al. The unique paradigm of spirochete motility and chemotaxis. Annu. Rev. Microbiol. 66, 349-370 (2012)

208. Bockenstedt, L. K., Liu, N., Schwartz, I. \& Fish, D. MyD88 deficiency enhances acquisition and transmission of Borrelia burgdorferi by Ixodes scapularis ticks. Infect. Immun. 74, 2154-2160 (2006).

209. Ouyang, Z., He, M., Oman, T., Yang, X. F. \& Norgard, M. V. A manganese transporter, BB0219 (BmtA), is required for virulence by the Lyme disease spirochete, Borrelia burgdorferi. Proc. Natl Acad. Sci. USA 106, 3449-3454 (2009).

210. Christodoulides, A., Boyadjian, A. \& Kelesidis, T. Spirochetal lipoproteins and immune evasion. Front. Immunol. 8, 364 (2017)

211. Lone, A. G. \& Bankhead, T. The borrelia burgdorferi VIsE lipoprotein prevents antibody binding to an arthritis-related surface antigen. Cell Rep. 30, 3663-3670.e5 (2020).

212. Verhey, T. B., Castellanos, M. \& Chaconas, G. Antigenic variation in the Lyme spirochete: insights into recombinational switching with a suggested role for error-prone repair. Cell Rep. 23, 2595-2605 (2018).

\section{Acknowledgements}

This work was supported by grants from the NIH (AI1 26033 and Al138949) and the Steven and Alexandra Cohen Foundation. E.F. is an investigator with the Howard Hughes Medical Institute. C.K. is supported by an $\mathrm{NIH}$ immunohematopathology research training grant (T32HL007974).

\section{Author contributions}

E.F., C.K., G.E.L. and S.N. researched data for the article and wrote the article. All authors contributed to discussion of the content and reviewed and edited the manuscript before submission.

\section{Competing interests}

The authors declare no competing interests.

Publisher's note

Springer Nature remains neutral with regard to jurisdictional claims in published maps and institutional affiliations.

(c) Springer Nature Limited 2020 